\title{
A review - polymer architectures via mass spectrometry and hyphenated techniques
}

Sarah Crotty, ${ }^{\mathrm{a}, \mathrm{b}}$ Selim Gerişlioğlu, ${ }^{\mathrm{c}}$ Kevin Endres, ${ }^{\mathrm{d}}$ Chrys Wesdemiotis, ${ }^{\mathrm{c}, \mathrm{d}_{*}}$ Ulrich S. Schubert, ${ }^{\mathrm{a}, b_{*}}$

(1)

${ }^{a}$ Laboratory of Organic and Macromolecular Chemistry (IOMC), Friedrich Schiller University Jena, Humboldstr. 10, 07743 Jena, Germany

b Jena Center for Soft Matter (JCSM), Friedrich Schiller University Jena, Philosophenweg 7, 07743 Jena, Germany

\author{
${ }^{\mathrm{c}}$ Department of Chemistry, The University of Akron, Akron, OH 44325, USA \\ ${ }^{\mathrm{d}}$ Department of Polymer Science, The University of Akron, Akron, OH 44325, USA
}

Article Info

Article History

Received

Accepted

Available online 
Keywords:

Mass spectrometry, liquid chromatography, ion mobility, tandem mass spectrometry, polymers, hyphenation.

\section{Abstract}

This review covers the application of mass spectrometry (MS) and its hyphenated techniques to synthetic polymers of varying architectural complexities. The synthetic polymers are discussed as according to their architectural complexity from linear homopolymers and copolymers to stars, dendrimers, cyclic copolymers and other polymers. MS and tandem MS (MS/MS) has been extensively used for the analysis of synthetic polymers. However, the increase in structural or architectural complexity can result in analytical challenges that MS or MS/MS cannot overcome alone. Hyphenation to MS with different chromatographic techniques $(2 \mathrm{D} \times \mathrm{LC}, \mathrm{SEC}, \mathrm{HPLC}$ etc.), utilization of other ionization methods (APCI, DESI etc.) and various mass analyzers (FT-ICR, quadrupole, time-of-flight, ion trap etc.) are applied to overcome these challenges and achieve more detailed structural characterizations of complex polymeric systems. In addition, computational methods (software: MassChrom2D, COCONUT, 2D maps etc.) have also reached polymer science to facilitate and accelerate data interpretation. Developments in technology and the comprehension of different polymer classes with diverse architectures have significantly improved, which allow for smart polymer designs to be examined and advanced. We present specific examples covering diverse analytical aspects as well as forthcoming prospects in polymer science.

\section{Contents}

1. Introduction

2. First dimension of complexity: linear homopolymers with MS 
2.1. Thermal-MS techniques

2.2. MS/MS techniques

3. First dimension of complexity: linear homopolymers with hyphenated techniques

$$
\text { 3.1. LC-MS based techniques }
$$

3.2. FFF-MS techniques

3.3. IM-MS techniques

3.4. Computational methods

4. Second dimension of complexity: linear copolymers

4.1. Block copolymers

4.1.1. Direct MS techniques

4.1.2. Thermal-MS techniques

4.1.3. MS/MS techniques

4.1.4. LC-MS and computational based techniques

4.2. Statistical copolymers

4.3. Random copolymers

5. Third dimension of complexity: complex polymers

5.1. Graft "like" polymers

5.2. Cyclic polymers

5.3. Star-shaped polymers

5.4. Branched polymers

5.4.1. Amidoamines and imines

5.4.2. Ethers and esters

5.4.3. Aromatic ring based

6. Conclusion

Acknowledgment 
References

77 Abbreviations

78 AUC Analytical ultracentrifuge

79 AF4 Asymmetric flow field-flow fractionation

80 APCI Atmospheric pressure chemical ionization

81 AROP Anionic ring opening polymerization

82 ASAP Atmospheric solid analysis probe

83 ATRP Atom transfer radical polymerization

84 CCS Collisional cross section

CE Capillary electrophoresis

86

CI Chemical ionization

87

CAD Collision activated dissociation

88

CZE Capillary zone electrophoresis

89
$Đ$
Dispersity

90

DESI Desorption electrospray ionization

91

DMSS Dimethylsilylstyrene

92

ECD Electron capture dissociation

93

EI Electron ionization

94

ELSD Evaporative light scattering detector

95

EO Ethylene oxide

96

ESI Electrospray ionization

97 ETD Electron transfer dissociation

98 FFF Field-flow fractionation

99 FT-ICR Fourier transform-ion cyclotron resonance

100 GC Gas chromatography 
101 HPLC High-performance liquid chromatography

102 ICR Ion cyclotron resonance

103 IM-MS Ion mobility-mass spectrometry

104 IR Infrared

105 ISD In-source decay

106 LAC Liquid adsorption chromatography

107 LACCC Liquid adsorption chromatography at critical conditions

108 LC Liquid chromatography

109 LDI Laser desorption ionization

110 LS Light scattering

111 MALDI Matrix-assisted laser desorption/ionization

112 MD Molecular dynamics

113 MS Mass spectrometry

114 MS/MS Tandem mass spectrometry

115 NMP Nitroxide-mediated radical polymerization

116 NMR Nuclear magnetic resonance

117 NP Normal phase

118 PAA Poly(acrylic acid)

119 PAMAM Poly(amidoamine)

120 PB Poly(butadiene)

121 PBS Poly(butadiene succinate)

122 PBT Poly(butylene terephthalate)

123 PCL Poly(caprolactone)

124 PDMS Poly(dimethylsiloxane)

125 PE Poly(ethylene) 
126 PEEK Poly(ether ether ketone)

127 PEI Poly(ethylenimine)

128 PEO Poly(ethylene oxide)

129 PET Poly(ethylene terephthalate)

130 PHEMA Poly(hydroxyethylmethacrylate)

131 PI Poly(isoprene)

132 PIB Poly(isobutylene)

133 PLA Poly(lactide)

134 PLGA Poly(lactic-co-glycolic acid)

135 PLUMS Polymer labeling using mass spectrometry

136 PMA Poly(methyl acrylate)

137 PMAA Poly(methacrylic acid)

138 PMMA Poly(methyl methacrylate)

139 PnBA Poly(n-butyl acrylate)

140 PO Propylene oxide

141 POM Poly(oxymethylene)

142 PP Polypropylene

143 PPG Poly(propylene glycol)

144 PPI Poly(propylene imine)

145 PPO Poly(propylene oxide)

146 PS Polystyrene

147 PSD Post-source decomposition

148 PSS Poly(styrene sulphonate)

$149 \quad \mathrm{P} t \mathrm{BA} \quad$ Poly(t-butyl acrylate)

150 PtBMA Poly(t-butyl methacrylate) 


\begin{tabular}{|c|c|c|}
\hline 151 & PVAc & Poly(vinyl acetate) \\
\hline 152 & PVAl & Poly(vinyl alcohol) \\
\hline 153 & PVP & poly( $N$-vinyl pyrrolidone $)$ \\
\hline 154 & $\mathrm{Py} / \mathrm{GC}$ & pyrolysis/gas chromatography \\
\hline 155 & Q & Quadrupole \\
\hline 156 & RAFT & Reversible addition-fragmentation chain transfer \\
\hline 157 & RI & Refractive index \\
\hline 158 & $\mathrm{RP}$ & Reversed phase \\
\hline 159 & RP-TGIC & Reverse phase-temperature gradient interaction chromatography \\
\hline 160 & SAXS & Small angle $\mathrm{X}$-ray scattering \\
\hline 161 & SEC & Size exclusion chromatography \\
\hline 162 & SG1 & $N$-tert-butyl- $N$-(1-diethylphosphono-2,2-dimethyl propyl) \\
\hline 163 & SID & Surface-induced dissociation \\
\hline 164 & SIMS & Secondary ionization mass spectrometry \\
\hline 165 & SORI & Sustained off resonance irradiation \\
\hline 166 & SL & Surface layer \\
\hline 167 & SPME/GC & Solid phase microextraction/gas chromatography \\
\hline 168 & TA-APGD & Thermal-assisted atmospheric pressure glow discharge \\
\hline 169 & ThFFF & Thermal field-flow fractionation \\
\hline 170 & TMPO & Trimethylolpropaneoxetane \\
\hline 171 & $\mathrm{ToF}$ & Time-of-flight \\
\hline 172 & UV & Ultraviolet \\
\hline
\end{tabular}




\section{Introduction}

Wherever we look in everyday life, we see polymers in a multitude of forms, e.g. as simple plastic bags, as packing material, as molded forms in toys, computers, seats, pens, in cars, trains, bikes, in shampoo and clothes, various household furniture, windows and in the paint on the wall. Nonetheless, polymers do not exclusively satisfy basic requirements; but high-tech materials are designed from polymers to challenge societal problems in medicine, energy generation and storage nowadays.

Depending on the application of the material (for example, polystyrenes as applied in construction and foam material or polylactides for the use in drug delivery systems), structurally distinct polymers with different macroscopic properties are prepared from a large library of structurally diverse monomers. This first level of structural complexity is not only influenced by the chemical nature of the monomer, but also by the length of the macromolecules and their molar mass distribution, expressed in the dispersity (Đ), as well as their end groups. By using two or more monomers, a second degree of structural complexity is reached for copolymers. Tuning the molar ratio of the monomers and their organization, such as in block, gradient or random copolymers, plays an important role in the resulting material having different chemical and / or physical properties. A third stage of structural complexity is reached by the arrangement of the monomers into a cyclic, star-shaped, combshaped or dendritic structure (Figure 1). These different architectures enable the engineering of highly complex molecules for high-end applications, e.g. micelles, amphiphilic block copolymers, polyion complexes, which encapsulate or entrap agents for tumor targeting applications [1]. 
197

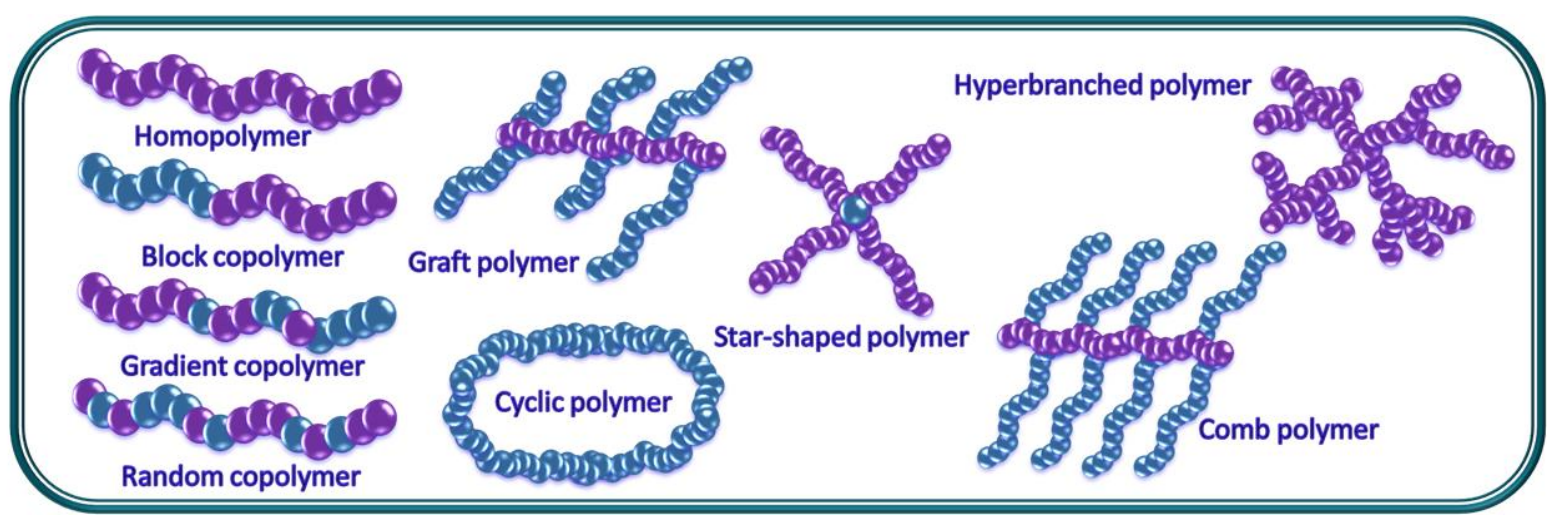

Figure 1. Schematic overview of various (co)polymer architectures.

However, by increasing the structural or architectural complexity of the polymeric system, the construction of the desired features results in far more demanding synthetic procedures, which increase the probability of defects. On the other hand, high-tech applications require well-defined and very reproducible products. To solve these opposing exigencies of synthesis reality and application requirements, a thorough and profound characterization of the polymer's architecture is required.

Mass spectrometry (MS) and tandem mass spectrometry (MS/MS) are extensively used to provide detailed information on structural properties of polymers [2-7]. Furthermore, many techniques that can be hyphenated to MS can provide greater insight on the polymer architectural information [8]. In particular, high-performance liquid chromatography (HPLC) techniques such as normal and reverse-phase HPLC and size exclusion chromatography (SEC) systems provide preseparation of complex mixtures and highly disperse samples for MS analysis $[2,4,5,7,9]$. Liquid adsorption chromatography at critical conditions (LACCC) is a specific HPLC condition, which enables the differentiation of end groups irrespective of the molar mass. First, the critical conditions are determined with a minimum of three polymer standards with the same end groups, but different molar masses and dissolved in a "good solvent". Then the "bad" solvent is added step by step, therefore promoting the elution of the 
standards from SEC towards adsorption mode. The critical conditions are reached when all standards have the same retention time. In addition to one-dimensional separation, 2D-liquid chromatography (LC) is utilized by combining multiple chromatographic techniques to separate samples by their inherent chemical and physical properties prior to MS. Moreover, the shape of a polymer can be investigated by field-flow fractionation (FFF), and although it is ideal for the separation of higher molar mass polymers, its connection to MS is not exploited extensively [10]. A more recently applied technique to polymers is ion mobilitymass spectrometry (IM-MS), which shows great promise because of its ability to separate gasphase ions depending on their shape, size and charge state [11]. The main advantages of IMMS are the ability to rapidly separate and differentiate isobaric and isomeric species without the need for MS/MS. Finally, in addition to hyphenated techniques, computational methods are being employed increasingly in polymer science for detailed data interpretation essentially facilitating the spectral analysis considerably [12].

Within this feature, MS techniques and hyphenated MS setups that were used to acquire information on macromolecular architectures are discussed in view of their benefit and disadvantages, as well as the desired future developments needed for obtaining more detailed insight on the molecular composition and the structure of polymers.

\section{First dimension of complexity: linear homopolymers with MS}

The complexity of homopolymers is mainly determined by the chemical identity of the monomer, the length of the polymer chains, its $Đ$ value and the $\alpha$ - and $\omega$-end groups, which might show an immense diversity depending on the applied synthesis technique. Additionally, the analyses of homopolymers is seen as the fundamental starting point for studies striving to develop and improve methods for more complex structures, to create libraries, facilitate predictions and enable interpretations of more complex data sets. In essence, the lengths of the 
polymer chains and $Đ$ values can be acquired by a one-dimensional MS technique. By degradation or fragmentation of the polymer, information regarding the end groups as well as the chemical nature of the repeating unit can be obtained $[3,13]$.

\subsection{Thermal-MS techniques}

Thermal-MS based techniques have the ability to decompose polymers by heat [13]. Subsequently, decomposed materials are analyzed by the mass spectrometer to obtain information regarding synthetic defects in the polymer chain and degradation products of the material. Several co-workers have used diverse techniques to investigate the polymer structure and pyrolysis products. For example, Zhang et al. utilized thermal-assisted atmospheric pressure glow discharge (TA-APGD) for structural characterization of polystyrene (PS) and poly(oxymethylene) (POM) [14]. The analysis of the degradation products by TA-APGD-MS provided information on the structural composition of the polymer without any sample pretreatment which can particularly be important for the analysis of polymer materials that are difficult to dissolve. Barton et al. used electrospray ionization (ESI)-MS in addition to matrixassisted laser desorption/ionization (MALDI)-MS to investigate the degradation mechanisms of poly(propylene oxide) (PPO) by heating the polymer prior to MS analysis [15]. One of the notable advantages of thermal methods is that it allows insoluble polymers to be analyzed by mass spectrometry. Tsai et al. used stepwise pyrolysis/gas chromatography (Py/GC)-MS method to investigate the thermal degradation of poly(aryl-ether-ether-ketone) PEEK. This study shows that Py/GC-MS can be a very useful analytical tool to separate and characterize, even the isomeric temperature dependent pyrolysis products by applying a stepwise temperature program [16]. Another application of thermal methods with mass spectrometry was on lacquer films. Lu et al. analyzed and compared the laccol components of natural and 
synthetic Rhus succedanea lacquer films, which also enabled the confirmation of the polymerization mechanism and structure of the macromolecule [17].

For linear homopolymers, thermal-MS techniques have mostly been used for determination of defects in microstructures and investigation of pyrolysis mechanisms since $1948[18,19]$. Characterization of the temperature dependent degradation products by thermalMS techniques can provide information regarding both physical and structural properties, thus helping the manufacturing of robust products.

\subsection{MS/MS techniques}

MS/MS techniques involve fragmentation of analyte ions in the mass spectrometer to obtain more detailed structural and/or architectural information. An MS/MS analysis can be carried out by using diverse scanning modes such as: product ion scan, precursor ion scan, neutral loss scan and selected reaction monitoring. Out of all these modes, the product ion scan is one of the most common MS/MS modes used for structural characterization of various synthetic polymers. During this mode of MS/MS analysis, a precursor analyte ion is isolated and this is followed by its activation and fragmentation inside the mass spectrometer. Finally, all of the fragmentation products are scanned and analyzed for more detailed investigation of the precursor ion structure. Many activation methods have been developed, such as collision activated dissociation (CAD), surface-induced dissociation (SID), photodissociation, electron capture dissociation (ECD), electron transfer dissociation (ETD), and post-source decay (PSD). Although numerous fragmentation techniques exist and are applied to synthetic polymers, the most widely used technique is CAD, which is closely related to PSD and insource decay (ISD) techniques. However, it does not always suffice for the full characterization of polymer architectures. Applications of MS/MS to a wide variety of 
polymer types and their fragmentation mechanisms have been discussed in detail in a review by Wesdemiotis et al. and several representative examples of such work are highlighted below [20].

For example, aromatic side chain based polymers such as PS has numerous different applications due to their importance in life science, and poly(2-vinylpyridine) (P2VP), for instance, in membranes as well as in batteries, and features a similar structure to PS, with only a slightly different side chain [20]. Furthermore, poly(acrylate)s are also important for contact lenses, membranes [21] as well as nanoparticles for energy storage [22]; MS/MS studies have been reported on poly(methyl acrylate) (PMA) [20,23], poly(methyl methacrylate) (PMMA) [20,24,25], poly(t-butyl methacrylate) (PtBMA), poly(t-butyl acrylate) $\mathrm{P} t \mathrm{BA}$ and $\operatorname{poly}(n$-butyl acrylate) (PnBA) [20,26]. Other important polymers that have been investigated include poly(vinyl acetate) (PVA) [20,27], poly(methacrylic acid) (PMAA) and poly(acrylic acid) (PAA) [20,28], polyesters [29-34], poly(lactide) (PLA) [35-38], poly(ethylene oxide) (PEO) $[20,39,40]$, and poly(oxazoline)s [41-44], all of which show remarkable promise in biodegradable and biocompatible materials [21,45-47]. Beyond these examples, many other polymer classes have been investigated under diverse ionization and fragmentation techniques, including poly(ethylenimine)s (PEI) [48,49], poly(dimethylsiloxane)s (PDMS) [20,39], complexes of PMMA with polyglycine [20], polyisocyanates [50], polyisobutylenes [51], and $N$-isopropylacrylamides [52], poly(ethylene/butylene terephthalate)s [29,30], polysulfide [53], polysulfones [54], polyesteramides [55], etc..

The above mentioned studies illustrate that, the differentiation between a pure polymer and its side products is a complex issue. MS/MS for homopolymers can be a very useful method for structural identification of the end groups and the sequence, i.e. the detection of defects in the polymer. Additionally MS/MS can also be used for determination of isobaric and isomeric species, and differences within a mixture of linear and nonlinear systems. 
However, in such cases, preseparation by hyphenated techniques might be necessary. Despite the diversity of the fragmentation techniques that can be used, MS/MS might not be enough to provide the necessary information to fully distinguish architectural differences. The use of LC as an analytic technique in the liquid phase prior to ionization enables the separation of polymer mixtures due to differences in polarity or hydrophobicity, which can provide complementary information and simplify the MS/MS data obtained from mixtures. Also, ion mobility (IM) spectrometry can provide additionally gas-phase separation of polymers before and/or after fragmentation. The combination of MS/MS with either one of these techniques makes it possible to obtain more detailed information on the nature of the end groups, presence of isomeric architectures, monomer sequences and degree of substitutions. However, these hyphenated techniques are mostly applicable on oligomers with a $m / z$ around 4000; thus, MS hyphenation techniques have to progress towards materials with higher molar masses and high dispersity.

\section{First dimension of complexity: linear homopolymers with hyphenated techniques}

\subsection{LC-MS based techniques}

Traditional analytical techniques for polymer characterization are pyrolysis MS, SEC and nuclear magnetic resonance (NMR) spectroscopy. The direct injection of a sample into the mass spectrometer without preseparation, known as direct MS, has been used as an analytical technique for polymers as well, but it has been proven that there are some cases where MS alone is insufficient for a comprehensive characterization of end groups, copolymer composition sequences, Đ etc. Thus techniques involving hyphenation with HPLC either separating by polarity or size and 2D-LC have been developed to provide a more detailed polymer characterization [8]. The hyphenation to MS is a versatile tool and many different types of LC-MS techniques were introduced over the past 20 years making LC-MS 
hyphenated methods important in polymer characterization and empowering them for sophisticated polymer analysis [8].

Many different 1D-LC systems hyphenated with MS are reported, particularly, for optimizing the transfer of the sample from a chromatographic system to the mass spectrometer. The main advantage of ESI is its compatibility with continuous hyphenation to diverse HPLC modes in comparison to MALDI, where most of the hyphenation techniques are carried out offline. Different hyphenations and different detectors are used to obtain extra knowledge regarding polymers, such as chemical heterogeneity and differentiation of (isomeric) architectures, thus enabling the chemist to improve the synthetic routes. A solvent free technique with an automated system was established for analyzing PEO under LACCC using a dry spraying technique [56]. PEO mixtures with different molar masses and different end groups were mixed and fractionated by using the LACCC technique prior to analysis of each fraction by solvent-free MALDI-MS. This tool allows an offline hyphenation of MALDI-MS with LACCC with much more convenient sample preparation procedure. Different spotting and simultaneous multisample deposition techniques were used to automate this hyphenation. Three different analytical methods have been combined to analyze PMMA homopolymers having different end groups [57]. LACCC-ESI MS with NMR spectroscopy and titration were used to investigate end group heterogeneity. Quantification of the components in the mixture was completed using an evaporative light scattering detector (ELSD) and identification of the different species was carried out via MS. Additionally, NMR spectroscopy was utilized for investigating the composition and titration for the quantification of $\mathrm{OH}$ groups. These complementary methods help to validate both structures and composition. The group of Barner-Kowollik hyphenated SEC with ESI-MS to analyze synthetic polymers that showed chromatographic broadening [58]. This hyphenation enables the determination of the structure via MS and of the molar masses via the refractive indices 
(RIs), as shown for PMMA oligomers used for calibration standards. SEC is widely used for molar mass determination of synthesized polymers; in this case band broadening was corrected through an in-house algorithm built for both detectors. This led to several observations: for lower molar masses $(<7 \mathrm{kDa})$, weak band broadening is present; however, with the algorithm, band broadening for $10 \mathrm{kDa}$ PMMA is corrected successfully. The resulting values were also in fair agreement with the manufacturer's value within an error of 15\%. In conclusion, the method shows consistency between the two detectors. Since conventional calibrations can be false, multi-detection is critical and important. Furthermore, in a follow-up contribution from the same group, important synthetic facts for tuning a polymerization, such as propagation rate coefficients $\left(\mathrm{k}_{\mathrm{p}}\right)$, were elucidated through pulsed laser polymerization and further analyzed via SEC-ESI MS [59]. This was found to be an accurate way to determine the molar mass of a polymer of any class, provided the polymer is ionizable by ESI. Propagation rates can be measured by such experiments which are important determinants for polymer chain lengths. The reversible addition-fragmentation chain transfer (RAFT) polymerization technique is used widely by many polymer groups in the world due to its facile 'one pot' polymer synthesis ability and the availability of a wide range of monomers that are capable of reacting. A series of acrylates were prepared using different initiators, and the reaction rates of the systems were investigated via SEC-ESI MS. The results were also compared with the predictions of the polymer model PREDICI ${ }^{\circledR}[60]$. Furthermore, CAD was used to analyze the intermediate reaction mixtures to obtain information concerning end groups and macromolecular structures. MS is essential for the investigation of polymer propagation and for establishing whether the proposed transformations occur. SEC-ESI MS followed by the application of the PREDICI ${ }^{\circledR}$ simulation tool is also used to determine the cross termination reactions in the RAFT polymerization of acrylates [61].

A 2D $($ RP-LC $\times$ SEC) chromatography system can also be coupled to MALDI and ESI. 
Even if this cannot be an online hyphenation, the procedure is still fully automated and very versatile. The RP-LC $\times$ SEC combination alone proves to be one of the most important analytical methods when specific conditions are applied in the first dimension, for example, LACCC. Linear poly(caprolactone) PCL with high and low molar masses were investigated by LACCC to identify end group heterogeneity, and the SEC dimension was used for molar mass separation [62]. As a result of hyphenating this technique to ESI and MALDI, the MS data served as the third dimension. High ionization efficiencies and multiply charged ion distributions were observed in ESI-MS spectra of the investigated PCL samples. Whereas in MALDI, prominently singly charged species, with low abundance, were observed because of the lower ionization efficiency and matrix interference. Pretorius et al. conducted a comprehensive RP-LC $\times$ SEC analysis to investigate the relationship between the chemical composition and molecular mass distributions of model phthalic anhydride / propylene glycol polyesters synthesized with a 30\% molar excess of glycol [63]. Gradient RP-LC allowed the isolation of homogenous fractions based on their chemical composition, which were then transferred to SEC for separation by hydrodynamic volume (Figure 2). The 2D (RP-LC $\times$ SEC) contour plots show that the samples have different $M_{\mathrm{n}}$ and $M_{\mathrm{w}}$ values: s23 $(135,216)$, s25 $(283,837)$, s28 $(569,1520)$, respectively. The numbers shown are peaks associated with the one-dimensional gradient RP-LC analysis. Furthermore, each fraction was also analyzed with MALDI-ToF MS to elucidate the various stages of the polyesterification reaction in terms of molar mass, chemical composition, and end groups [63]Error! Reference source not found. 

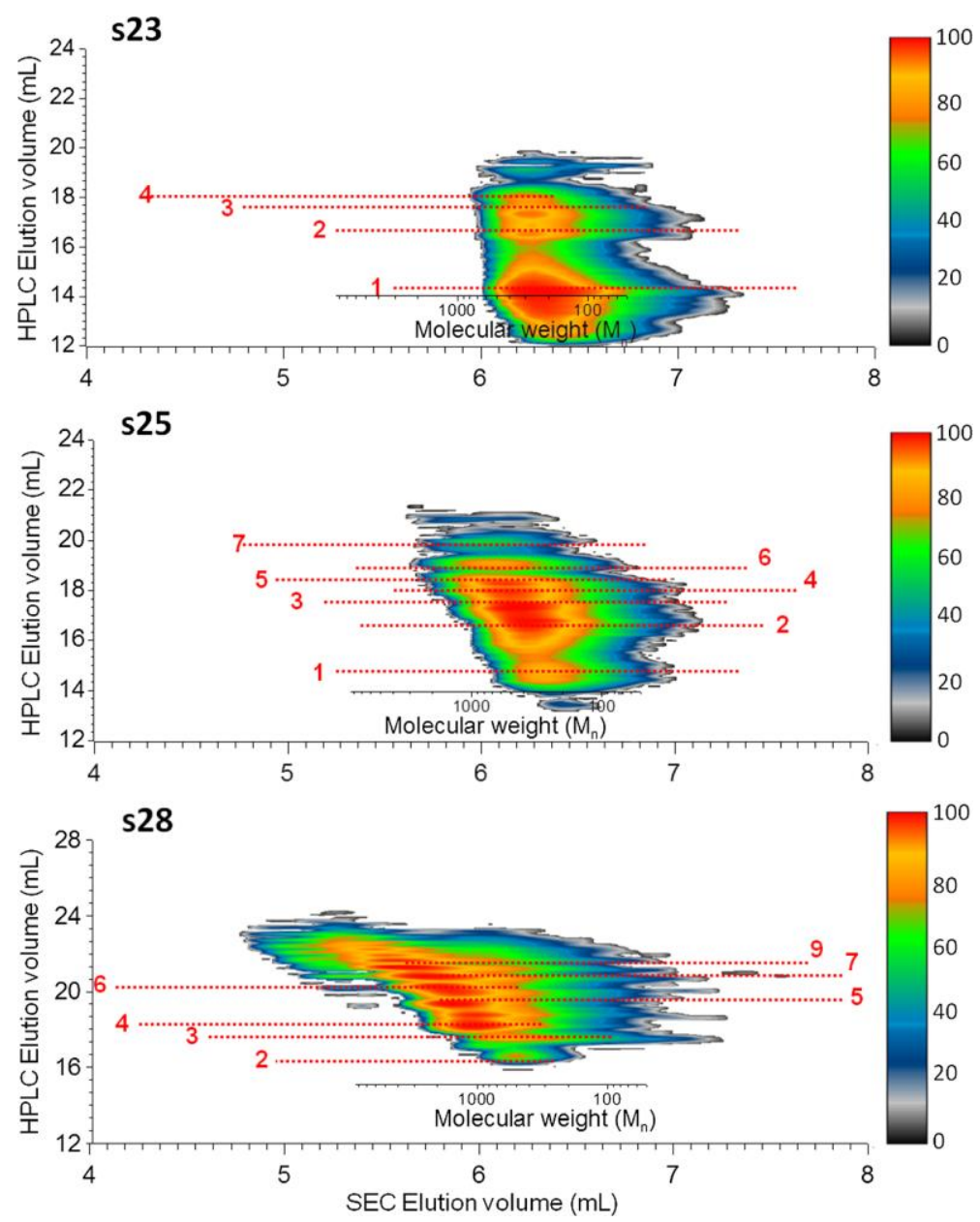

413 Figure 2. 2D (RP-LC $\times$ SEC) contour plots of polyester samples: s23, s25, s28. Reproduced 414 from Ref. [63].

Depending on both chemical and physical properties of polymers, the RP-LC $\times$ SEC 417 technique's ability of two-dimensional separation demonstrates its versatility, which makes it a very sophisticated and useful tool for polymer analysis. Therefore, its hyphenation to mass spectrometry can provide many advantages for polymer analysis, most importantly reducing

420 the complexity of analysis of a highly heterogeneous polymer mixture having high dispersity:

421 it will promote technology and insight for chemists to further develop smarter polymer 422 designs. 


\subsection{Field-Flow Fractionation (FFF)-MS techniques}

Field-flow fractionation is a separation technique, used mostly for aqueous solutions of disperse and charged or neutral species, dependent upon differing velocities in a field [64]. FFF is used for synthetic polymers but is less commonly applied in combination with the MS technique. Coupling this technique to a mass spectrometer is challenging due to the high molar masses of the investigated materials. Hassellöv et al. reported the analysis of low molar mass poly(styrene sulphonate) (PSS) and PEO standards by FFF-ESI MS [65]. After the low molar mass polymers were separated by FFF, they were analyzed by ESI MS to obtain compositional information. Further studies to obtain end group compositions were carried out by applying MS/MS. However, the technical online hyphenation was also highlighted and high salt concentrations caused clogging of the ESI-MS and high background signals for an efficient separation. Another example for the combination of FFF with MS was the coupling of a thermal field-flow fractionation (ThFFF) setup to MALDI-ToF MS for the analysis of high molar mass PS standards [10]. ThFFF enabled the separation and the fractionation of polymers that were further analyzed by MALDI-ToF MS. After the separation and analysis with both ThFFF and MALDI-ToF MS, it was shown that both techniques have correlating results; in addition, FFF confirmed the macromolecular conformations. The resolution obtained was $<15 \mathrm{kDa}$ for the ThFFF separation and a maximum molar mass of $575 \mathrm{kDa}$ by MALDI-ToF MS. To conclude, FFF is a promising technique for obtaining architectural information such as conformation and size of complex polymeric systems. Furthermore, the FFF-MS hyphenation shows that numerous dimensions expand the comprehensive characterization of polymers. This particular hyphenation needs further improvements and additional applications, specifically for more complex architectures of synthetic polymers where it is essential to characterize the composition and conformation. 


\subsection{Ion Mobility-Mass Spectrometry (IM-MS) techniques}

Previously used for 'omics', IM-MS has recently been applied to polymers and metallo(supramolecular) materials [11], and its applications to polymer science continue to increase. IM spectrometry provides an additional dimension for elucidating different conformations or architectures present in an analyte. With IM-MS, gas-phase ions are separated according to their mobility and composition. Information obtained by IM separation can be used to render collision cross-sections, which relate directly to the macromolecular shape.

Isobaric ions, which are two different chemical species having the same nominal mass (mass difference at ppm level) but different elemental composition, are often encountered in polymer analysis and can only be resolved with high resolution spectra. If isobaric species are not resolved, obtaining a more detailed structural characterization with MS/MS experiments can be very difficult. This can result in very complicated MS/MS data, having fragment ions from both species in the same spectrum. Preseparation by a LC technique might be an option for a situation such as this. However, the most important advantage of IM over LC techniques is that the separation occurs within milliseconds in the gas phase, and does not require excessive solvent usage. IM separation is an ideal technique for hyphenation to MS since the separation takes place in the gas phase. Isobaric species can be separated depending on conformational or architectural differences at a much faster rate in the gas phase. After IM separation, these isobaric species can further be investigated separately by MS/MS for more detailed structural characterization, if needed. Furthermore, having higher resolution in IMMS/MS (for example by employing $\mathrm{Q} / \mathrm{ToF}$ instrumentation) enables better mass accuracy in MS/MS studies. For example, Hilton et al. were able to investigate a poly(ethylene glycol) (PEG)s mixture composed of two PEG chains having different end groups by IM-MS/MS [66]. In this particular example, these two isobaric PEG chains have diol and monooleate end 
groups, and result in the same nominal $\mathrm{m} / \mathrm{z}$ values. However, the structural difference at the

475 end group of these two chains results in two distinct drift time values in the IM spectrum.

476 Separation was therefore accomplished before MS/MS analysis. Figure 3 shows the IM

477 separation of these two chains at an isolated $\mathrm{m} / \mathrm{z}$ value of 553 . Further MS/MS

478 characterization of these separated PEG chains reveals the structural difference at the end

479 groups (Figure 3). One can see that IM-MS could separate polymers having different end groups from both PEGs in the millisecond range when compared to LACCC, which requires a

481

482

483

484

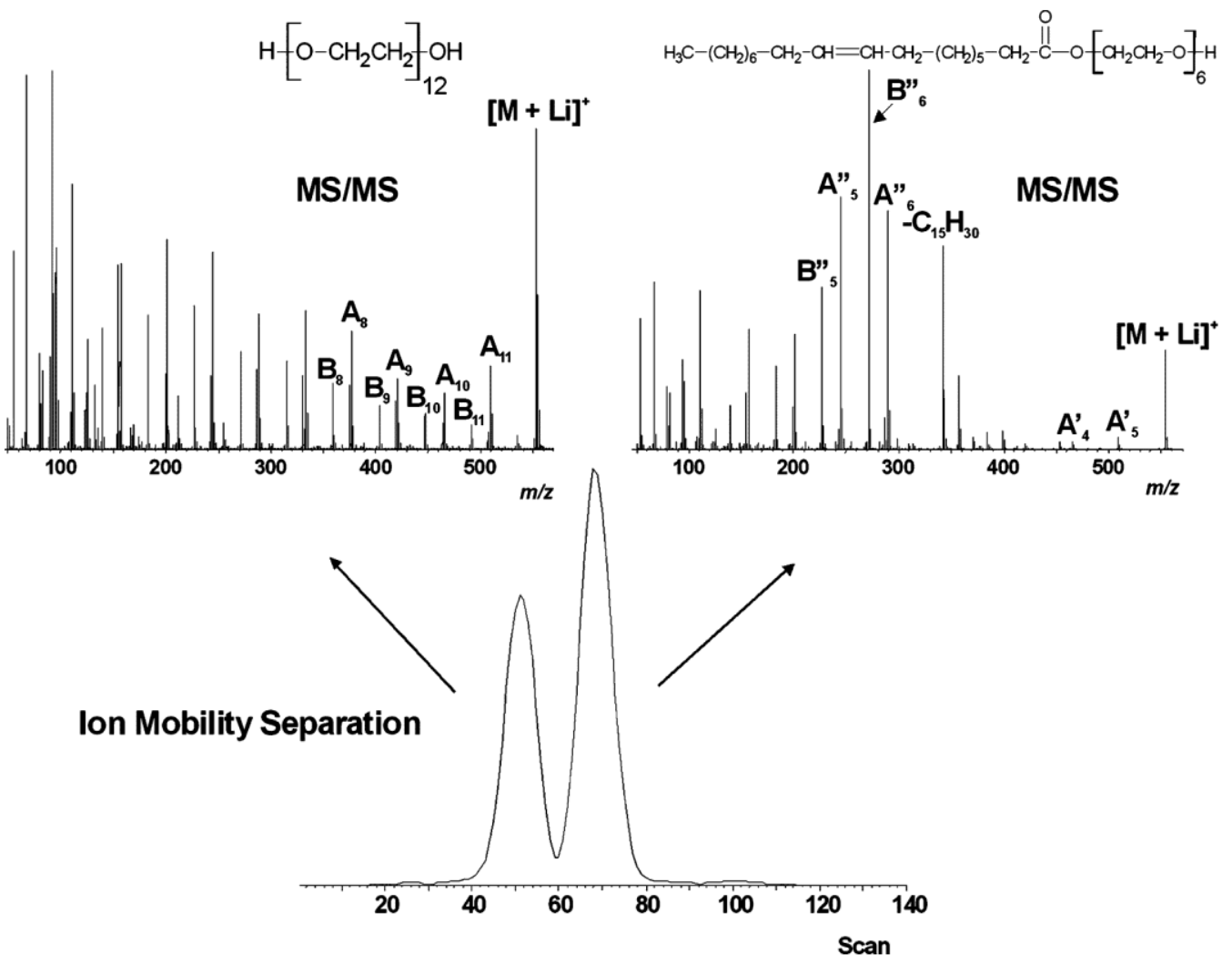

485

486

Figure 3. Arrival time distribution (bottom) of $\mathrm{m} / \mathrm{z} 553$ from a mixture of PEG 1000 and PEG 
monooleate and IM-MS/MS spectra (top) from the two peaks noted. Peaks in the IM-MS/MS spectra are partially annotated. IM-MS/MS spectra are very similar to those noted from MS/MS data (without IM separation) of the same oligomers, obtained from PEG 1000 and PEG monooleate separately. Reproduced from Ref. [66].

Synthetic inorganic polymers, such as polysiloxane and polyphosphazene based polymers, were investigated with ESI-IM-MS by Scionti et al. [67]. IM-MS provided information on the existence of higher order assemblies for the polysiloxanes. It also confirmed that the reaction of $\mathrm{NH}_{4} \mathrm{Cl}$ with $\mathrm{PCl}_{5}$ under aerobic conditions produces poly(dichlorophosphazene)s with both tadpole as well as linear architectures.

Waters being the manufacturer of the first commercial IM-MS instruments (Synapt line of models) published a report on the analysis of polymers with IM-MS and MS/MS. Compared to traditional MS techniques, added IM dimension, shown to facilitate the determination of physical properties and sequences [68]. Kim et al. applied ESI-IM-MS combined with molecular dynamics (MD) simulations on PLAs having different stereoregularities, formed from cyclic lactide dimers by ring-opening polymerization [69]. The gas-phase conformation of PLAs is affected by stereoregularity and architecture (linear or cyclic), both of which influence collision cross-section value trends. Poly-LD-lactide (PLDLA) has more structural flexibility in comparison to poly-L-lactide (PLLA), thus PLDLA is able to maximize intramolecular interactions, showing lower energies and higher degrees of weak hydrogen bond interactions, which lead to more compact structures for PLDLA with the exception of small macrocycles. These differences in stereoregularity result in different physical properties and structure. IM-MS in conjunction with computational analysis is a powerful tool to differentiate these structural differences. Therefore, it is expected that IM-MS analysis can be a promising technique to characterize polymers with different structures and 
stereoregularities. Trimpin et al. used IM-MS to characterize PEO polymers of relatively high molar masses using ESI as the ion source [70]. Simulated and experimental cross-sections were compared and evaluated. High charge states were found to have an open and dynamic conformation. The intrinsic limitation with ESI is the accessible molar mass range, which is less restricted with MALDI. These two different sources coupled to IM-MS are becoming increasingly more attractive for polymer chemists as a faster way to obtain dense and rich information on polymer architectures. IM-MS has also been used to provide supportive architectural information to ETD fragmentation patterns for linear polyester samples by obtaining collision cross-section values of the major fragment series [36]. Another type of polymer, poly(propylene) (PP), was investigated using pyrolysis with an atmospheric solid analysis probe (ASAP) and IM-MS [71]. Pyrolysis was used to decompose polymers and IMMS used to determine their $\mathrm{m} / \mathrm{z}$ values as well as to separate the polymer from any additives. The pyrolysis products were established and compared to prior studies. These two methods coupled to each other help to segregate species and confirm their structure. Barrère et al. showed that ASAP-IM-MS can be used to successfully distinguish between polyester and polyethylene blends [72]. ASAP as an ionization source is an effective technique for the characterization of polymer blends with different polarities without the need of complex sample preparation, which is particularly useful for samples that are difficult to dissolve. Therefore, the combination of ASAP and the structural separation provided by IM allows the clear identification of relatively large pyrolysis products from different polymers such as in PLA-polyethylene (PE) blends. Song et al. reported a detailed study using ESI-IM-MS on a PMMA homopolymer prepared by radical polymerization [73]. The small end group differences in the resulting PMMA system, could be discriminated by IM without the need of a time consuming LC separation. Finally, Hoskins et al. used IM-MS to differentiate between linear and cyclic PCL polymers [74]. The study shows that the IM step is capable of 
separating linear and cyclic architectures in a blend since the individual structures result in different drift time values (Figure 4). Figure 4c also displays that ions having same $\mathrm{m} / \mathrm{z}$ values in mass spectrum were differentiated clearly according to their architectural differences.

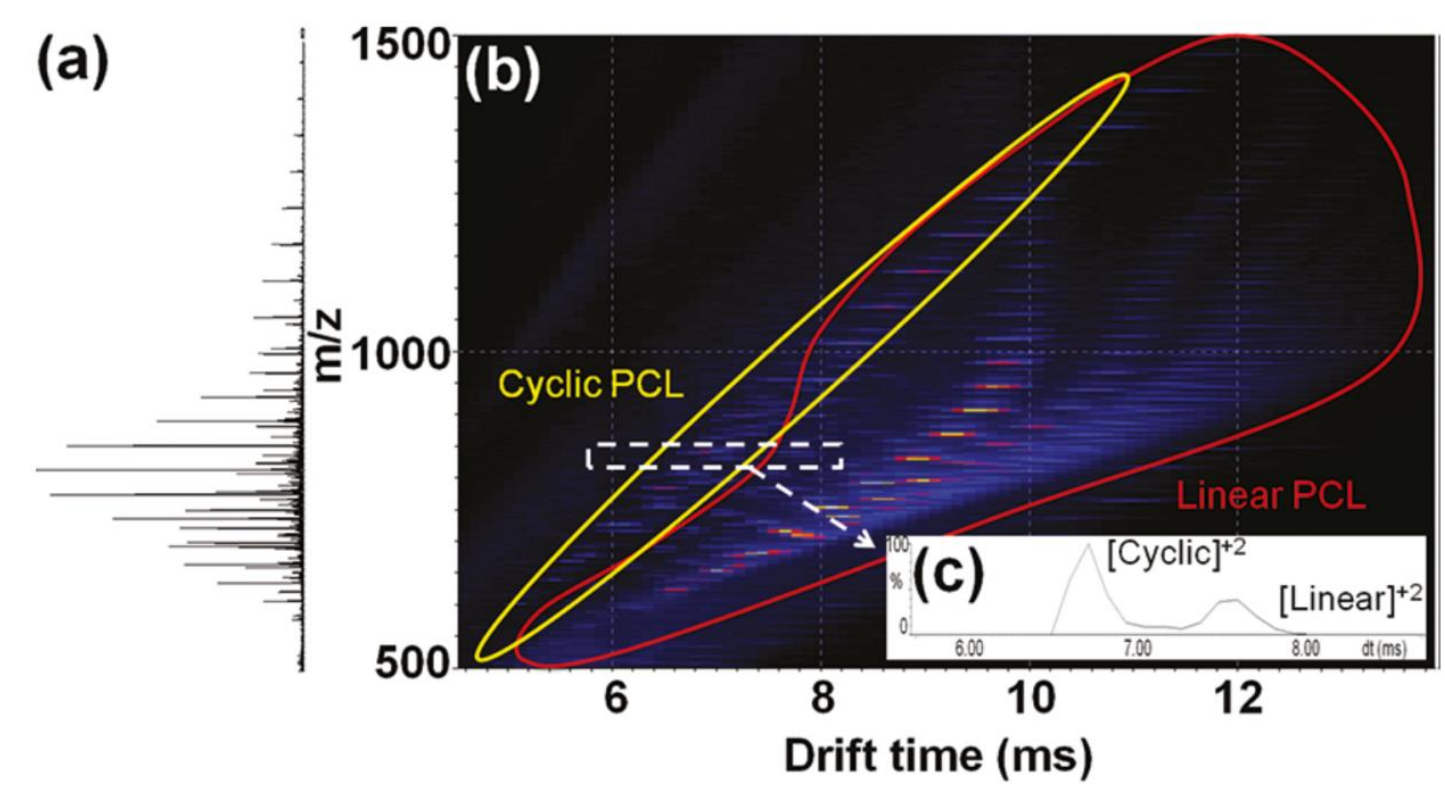

Figure 4. Mass spectrum (a) and IM-MS 2-D plot (b) of a PCL blend having both cyclic and linear structures. The intensities of the ions detected are incorporated as a false color plot with red as the most abundant and blue as the least abundant ions. The inset (c) shows the drift time scale of a separated peak at $893.13 \mathrm{~m} / \mathrm{z}$. Reproduced from Ref. [74].

All the reported examples show that, as a hyphenated technique, IM-MS can be a very valuable analytical technique for polymer science as it already is for 'omics' fields. Its capability of differentiating architectural differences in the gas phase provides an advantage over other chromatographic techniques and makes it a very promising hyphenated technique for solving current and future analytical problems that can be faced during the characterization of complex polymeric systems. 


\subsection{Computational methods}

Computational methods are becoming more prominent in facilitating the analysis of MS and MS/MS spectra. For example, different poly(hydroxyethylmethacrylate) (PHEMA) backbone structures were analyzed via ESI by Jackson et al. The data analysis was done by the Polymerator software (Figure 5 (a)) [75]. End group analysis was performed, and different fragmentation series were obtained and analyzed with the Polymerator software to identify polymer fragments (Figure 5 (b)). The Polymerator enabled an easier and faster interpretation of the MS/MS data. Moreover, the information obtained can also support the formation of future libraries of fragments.

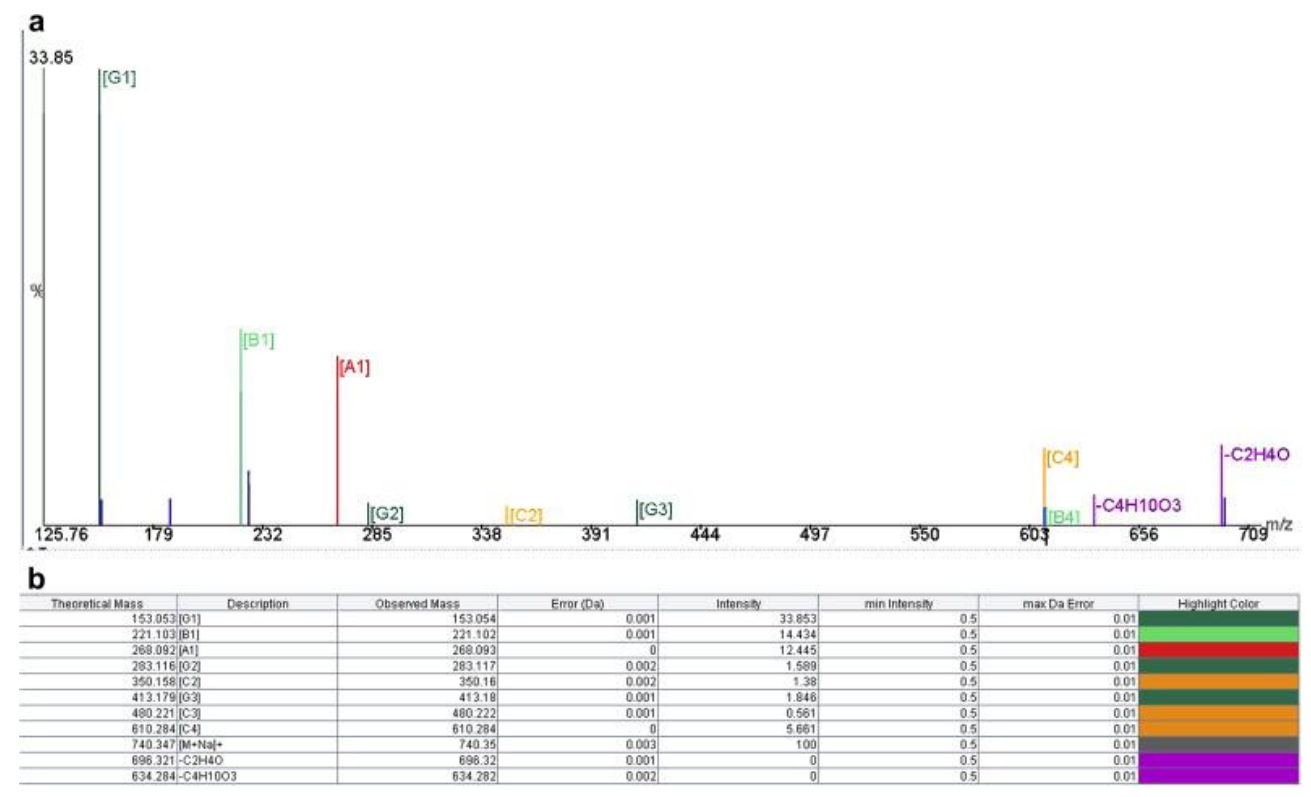

Figure 5. (a) Screenshot from Polymerator software of annotated ESI-MS/MS spectrum from the sodiated tetramer of PHEMA. (b) The details of the annotated fragment ions are displayed by the Polymerator software in the table. Reproduced from Ref. [75].

Another advantage of the Polymerator software is that it is dependent on the polymer class and the knowledge of individual fragments. This feature makes it possible to apply it to 
571 different polymers to allow the validation of manual and computed fragmentations [76].

572 Jackson et al. also used this software to distinguish between end group cleavage and the 573 fragmentation of the polymer chain, and performed detailed end group analysis of

574 poly(propylene glycol) (PPG) by ESI with low energy MS/MS [77]. Williams et al. used the Polymerator software to analyze the CAD spectra of PEO ions having different end groups and different cationization agents, formed by desorption electrospray ionization (DESI) [78]. The authors emphasize the ability to determine microstructures for 'polymers'. However, prior knowledge of the fragmentation mechanisms is a requirement for utilizing the software. On the other hand, software based analysis can benefit from polymer libraries and is quicker than manual interpretation. In addition to the Polymerator, the polymer labeling using mass spectrometry (PLUMS) software is another computational method developed for the interpretation of fragmentation spectra [79]. This software was used to determine the fragment series formed, knowing that no prior knowledge on the chemical behavior of the polymer class was required. Using PLUMS, practically all of the possible fragments were determined with an attached probability, in contrast to the Polymerator, where chemical parameters are pre-identified.

In conclusion, all discussed studies represent an immense step forward in polymer science in view of a detailed characterization of polymer architecture and conformation. The growth of these techniques will help chemists to evaluate complex polymers in a more in-depth manner by making them less time-consuming. 


\section{Second dimension of complexity: linear copolymers}

\subsection{Block copolymers}

Block copolymers of linear architecture are composed of at least two segments/domains bound together. Block copolymers may undergo microphase separation due to differences in the solubility of the different segments in a specific solvent. This results in different morphologies in solution, for example, the formation of micellar structures. Furthermore, the morphologies of the formed nanostructures can be tuned via the relative length of the individual blocks. Such types of polymers are widely used in the fields of drug delivery, thin films, membranes etc. [1,80]. The use of MS and specific hyphenations allow the determination of block lengths and sequences, as well as the differentiation between different architectures/microstructures [81].

\subsubsection{Direct MS techniques}

Direct MS analysis of polymers helps to elucidate side products, end groups and the degree of polymerization of each block. For example, Houshia et al. analyzed high molar mass block copolymers comprising of EO and PO segments via MALDI-FTMS [82]. The authors use correlation functions and probability functions to evaluate diverse copolymer components overlapping isotopic patterns, which is required for polymers having high molar masses where the loss of resolution is predominant and the certitude of copolymer composition becomes weak.

MS analyses are generally more complex for linear copolymers than for homopolymers because the ionization efficiency can significantly differ as a result of the different lengths and co-monomers structures in each block. Nonetheless, direct MS can provide elements of a comprehensive characterization of copolymers, though additional methods such as thermalMS, MS/MS and hyphenated techniques are needed for further elucidation of the architecture. 


\subsubsection{Thermal-MS based techniques}

Similarly to homopolymers, thermal degradation techniques are useful for accomplishing

621

622

623 detailed evaluations of block copolymer structures. For example, Zhang et al. used thermalassisted atmospheric pressure glow discharge mass spectrometry (TA-APGDMS) to evaluate block copolymer fragments [14]. The block copolymers were based on POM and poly(butadiene succinate) (PBS) segments. Firstly, fragmentation pathways were established for standard homopolymers, and later for the corresponding copolymers. Characteristic degradation patterns from the POM homopolymers were also observed in the POMcontaining copolymers; however, this did not hold for PBS homopolymers and its block copolymers. This study proved that thermal degradation is applicable to copolymers and helps to elucidate their structure and chemical composition. PEO- $b$-(PPO)- $b$-PEO triblock copolymers were also studied using thermal degradation [83]. SEC, NMR spectroscopy and MALDI-MS were used to characterize poloxamer 407. The products of heat induced degradation were analyzed by MALDI-MS and by solid phase microextraction/gas chromatography (SPME/GC) MS. These complementary methods are essential in revealing the oxidation mechanism of poloxamers and their structures. Ohran et al. have investigated three kinds of block copolymers: PS- $b$-P2VP, P2VP- $b$-PMMA and poly(isoprene) (PI)- $b-$ P2VP via direct pyrolysis-MS [84]. This method allows the evaluation of backbone cleavages and, as a consequence, the determination of the sequence and possible crosslinks formed.

Lattimer et al. investigated the pyrolysis products of segmented polyurethanes with MALDI-MS after thermal degradation. In this study, different temperatures were used during pyrolysis to investigate thermal degradation mechanisms by analyzing the pyrolysis products (up to $10000 \mathrm{Da}$ ) with MALDI-MS [85]. Whitson et al. characterized commercial polyurethanes by atmospheric pressure chemical ionization (APCI)-MS in combination with a 
643 direct insertion probe (DP). By slowly increasing the temperature, the thermal degradation 644 products and the other components in the blend, such as additives were successfully 645 separated. The temporal separation that took place in this method was based on compound 646 volatilities and bond stabilities. As a result, combining thermal method by using DP allowed 647 these insoluble materials to be directly analyzed by MS [86]. Another application of this 648 method was done on cross-linked amphiphilic conetworks composed by graft $\operatorname{copoly}(N, N$ 649 dimethyl acrylamide-g-dimethylsiloxane) (PDMAAm- $g$-PDMS) and their blends [87]. The 650 different compositions of hydrophobic and hydrophilic components in these graft copolymer 651 blends were distinguished by utilizing DP-APCI-MS. In addition to different comonomer 652 compositions, information on the thermal stability of the different domains within the 653 copolymer was obtained with the help of precise pyrolysis temperature control by DP [87].

In accordance with the studies discussed above, thermal-MS techniques have great 655 potential for providing important information on structural (block lengths, defects in

656

\subsubsection{MS/MS techniques}

MS/MS techniques have proven to be very useful for the investigation of sequences and block lengths of different copolymer structures. Cerda et al. subjected $[\mathrm{PPG}-b-\mathrm{PEG}]^{2+}$ to both ECD and CAD analysis, which allows for detailed structural characterization of this copolymer [40]. The product ions generated from the ECD experiments showed that all of the copolymers studied consist of diblock structures, and not triblock structures that are 
designated by the polymer manufacturer. Furthermore, Baumgaertel et al. analyzed different poly(2-alkyl-2-oxazoline) block copolymers (variation of the alkyl side group), via ESI-and MALDI-MS using CAD for fragmentation [88]. The usage of both ionization methods revealed detailed information regarding the side products, monomer sequence and block length. The fragmentation patterns of block copolymers in such studies are mostly derived by using prior knowledge of the behavior of the analogous homopolymers under the same MS/MS conditions. Crecelius et al. studied $m$ PEG- $b$-PS block copolymers prepared via atom transfer radical polymerization (ATRP) [89]. MALDI-ToF MS/MS was used for detailed structural characterization. For this reason, the homopolymers of each comonomer were investigated separately prior to the MS/MS analysis of the block copolymers to facilitate the interpretation of the fragmentation results. Detailed structural analysis by MALDI-MS/MS on these copolymer revealed accurate block lengths of PS and $m$ PEG. The authors claimed to observe only fragments from both blocks individually, thus, concluding that a scission between the blocks takes place. The main fragmentation mechanisms observed were 1,4hydrogen elimination and McLafferty rearrangement within the PEG chain.

In addition to $\mathrm{CAD}$, different ion activation methods, such as $\mathrm{ECD}$, can also provide useful MS/MS data for architectural analysis of copolymers. One of the earlier examples is the application of both CAD and ECD for sequence analysis of PEG- $b$-PPG- $b$-PEG copolymer mixtures. According to the results presented by Cerda et al., CAD can lead to misleading rearrangements, however, as mentioned before, ECD causes minimal rearrangements thus preventing internal fragment formation [90]. Therefore, extensive structural details such as sequence information on complex mixtures of low abundance block copolymers can be obtained by using ECD as an ion activation method for MS/MS. Another study that involves the comparison between $\mathrm{CAD}$ and ECD fragmentation patterns was performed on random and block polyacrylate and polyether copolymers [91]. The study shows that CAD alone is able to 
693 differentiate random and block copolymers. Random poly(methyl methacrylate)-r-poly $(n-$ 694 butyl methacrylate) (PMMA-r-PnBMA) oligomers tend to undergo random losses of MMA or 695 BMA monomeric units, but block poly(methyl methacrylate)- $b$-poly( $n$-butyl methacrylate)

696

697

698

699

700

701

702

703

704

705

706

707

708

709

710

711

712

713

714

715

716

717

(PMMA- $b$-PnBMA) oligomers lose BMA monomeric units preferentially [91].

Based on the examples portrayed, MS/MS provides essential details on structural properties of copolymeric systems such as comonomer sequences, microstructural differences, and block lengths. Accurate determination of such properties of copolymers is essential for the assessment of material's quality.

\subsubsection{LC-MS and computational based techniques}

As described previously, copolymers are rather heterogeneous and disperse systems. For copolymer structures of high complexity, analysis on additional dimensions may be essential for detailed characterization. Direct MS characterization is not always sufficient to elucidate the architecture of block copolymers. As a consequence, hyphenated analytical techniques have been employed to facilitate the analysis of such polymer systems.

The following selected examples highlight the successful characterization of block copolymers using 1D-LC-MS. Pyrolysis-GC-MS hyphenated to either a gradient reversedphase (RP) LC or a SEC instrument were used in a study by Kaal et al. on PEG- $b$-PPO copolymers [92]. While SEC or RP-LC separated the copolymers according to their hydrodynamic volume or polarity, respectively, the quantitative information for different compositions was obtained by pyrolysis-GC-MS. Furthermore, this method also permits the calculation of monomer feed ratios, which is important for a validation of the composition, as well. Leeuwen et al. analyzed block copolymers composed of $m$ PEO and PCL blocks via ESIMS and APCI MS combined with RP-LC fractionation [93]. Mass spectrometric analysis was performed both in the positive and negative mode. Further, gradient elution was applied for 
718 low molar mass copolymers and used to determine the block lengths as well as the comonomer composition. $m$ PEO- $b$-PCL was investigated under APCI ionization conditions as a complementary method to ESI and for hyphenation to a HPLC system in order to evaluate the block length of both species present in this biocompatible polymer that can form micellar structures. Hayen et al. applied RP-LC-ESI MS to poly(ether- $b$-ester) block copolymers, i.e. PEO- $b$-poly(butylene terephthalate) (PBT) [94]. Application of RP-LC prior to MS analysis provided a separation of the side products having different segments, but similar molar masses. Girod et al. analyzed PEO- $b$-PS block copolymers by hyphenating LACCC to ESIMS/MS [95]. The LACCC conditions were optimized by tuning the salt concentration in the mobile phase for better separation of the species with different end groups present in the sample, independent of their molar mass. Additionally, the length of the PS block was analyzed and also confirmed by CAD MS/MS. As a result, combination of these complementary methods allowed both the determination of the PS block length and end group analysis. A better understanding of RAFT polymerization of poly( $N$-vinyl pyrrolidone) (PVP) and poly(vinyl acetate) (PVAc) block copolymers (PVP- $b$-PVAc) and its byproducts was provided by Fandrich et al. who used gradient LC-MALDI and LC-Fourier transform-infrared (FT-IR) spectroscopy [96]. Hyphenation of various LC techniques to MALDI-MS and FT-IR made it possible to differentiate the main products from the by-products. Additionally, the results obtained throughout the study essentially helped to derive the possible changes in the reaction mechanism that could cause formation of copolymer blends containing long blocks of either PVP or PVAc.

Separation of compounds in complex mixtures can also be carried out by utilizing two different LC methods in combination to obtain two dimensional separation (2D-LC). 2D-LC is a time-consuming technique; however, it is a very informative method and can be effectively used for copolymer analysis. Baumgaertel et al. used 2D (LACCC $\times$ SEC) in 
743

744

745

746

747

combination with MALDI- and ESI-MS/MS for the analysis of poly(2-ethyl-2-oxazoline- $b$-2(2,6-difluorophenyl)-2-oxazoline) (p(EtOx- $b$-oDFOx) block copolymers, which have potential medicinal applications. In this study, the application of LACCC allowed separation of the polymers containing different end groups with varying polarity as well as the series with different block lengths. Further analysis of the LC fractions with MALDI-MS and ESIMS/MS allowed full structural characterization of PEtOx chain transfer and termination products. Finally, quantification of the separated components were carried out by addition of SEC dimension. The 2D contour plot in Figure 6 shows three fractions: fraction 1 corresponds to the PEtOx homopolymer with an ester end group (7.5 vol.\%), fraction 2 corresponds to the copolymer (p(EtOx-b-oDFOx) $(87$ vol. $\%)$ and fraction 3 corresponds to the PEtOx homopolymer with a hydroxyl end group (4.5 vol.\%) [97]. As a result, combination of the essential information obtained from all three methods was used to ensure that all products are comprehensively characterized. 
Other studies from the Weidner group involved imaging of similar copolymer

763

764

765 architectures by having specific RP-LC conditions hyphenated to a MALDI target via a spray to separate and identify different compositions within one copolymer [98]. Weidner et al. introduced this technique to study copolymers comprised of propylene oxide (PO) and ethylene oxide (EO) blocks, which contained isobaric species or species with very similar masses (within $2 \mathrm{Da}$ ). The separation of components differ in PPO and PEO content by LACCC could differentiate compositions from a sample that has overlapping $\mathrm{m} / \mathrm{z}$ values, hence composition specific calibration curves were constructed based on the number of PO and EO comonomers. Subsequently, more complex structures could be thoroughly separated, and characterized, thereby setting the foundation for the next step: sequencing of copolymer structures present in the blend.

Barqawi et al. used an automated system to deposit fractions of a triblock copolymers, that were separated in 2D LC/SEC run, onto a MALDI target [99]. Firstly, the critical conditions of poly(isobutylene) (PIB) homopolymers were identified, which was used as the first RP-LC dimension for the identification of the end group heterogeneity. In the second dimension, a SEC column was utilized to determine the molar mass of the polymer and to quantify the products as well as the undesired side products. The $2 \mathrm{D}-\mathrm{LC}$ eluents were mixed with the salt and the matrix solutions and then sprayed onto a MALDI target with the help of an automated transfer module. This whole automated system allowed a continuous MS data collection at the various 2D-LC elution volumes, which essentially yielded numerous time-dependent spectra. This system is a major achievement in automation and characterization of complex polymer samples due to the fact that the first two dimensions are hyphenated to a MALDI-MS. It also allows a facile way to interpret spectra due to less overlapping peaks, an estimation of composition, chain length, and architecture of symmetrical and non-symmetrical triblock 
copolymers. In a different offline technique 2D-LC was combined with MALDI-MS to analyze EO based block copolymers [100]. LACCC was used as the first dimension to separate polymers bearing different functionalities. The second dimension was either a SEC or a LAC system which gave evidence for the molar mass of all products present with varying functionality. The setup was hyphenated to MALDI-MS in an offline manner. Raw copolymer samples are very complex to analyze due to high heterogeneity, however, advanced 2D fractionation prior to MS analysis makes the characterization of architectural details possible. It should also be considered that, this is a time-consuming method since optimization of numerous factors (solvent, column, temperature etc.) for separation is needed.

Over the years, computational methods have grown and make the analysis of polymeric materials faster and more straightforward. Weidner et al. have extensively studied PEO- $b$ PPO-type block copolymers [101]. They have developed a software-based chromatographic separation of block copolymers that are subsequently analyzed via MALDI-ToF MS. MALDI mass spectra of complex copolymeric systems can result in overlapping isotope patterns of ions comprising different monomeric compositions in the mass spectra. Therefore, preseparation of these species prior to MS data collection become essential for more accurate determination of monomer composition. In this case PEO- $b$-PPO- $b$-PEO was investigated thoroughly, by first establishing the critical conditions of PPO which was followed by separation of the components with LAC according to their monomer compositions. All of the fractions obtained from the separation were then sprayed onto a MALDI target for MS analysis of each component. These MS data were then processed by the MassChrom2D algorithm and provided the number of EO and PO units present in each specific fraction. In addition, the intensity obtained from chromatographic data was inserted in the $2 \mathrm{D}$ composition plots formed by the MALDI spectra. The entire workflow of the protocol is 
summarized in Figure 7. This method is a major step forward in quantifying and identifying the copolymers with different monomeric compositions present in complex mixtures.



Figure 7. Scheme of measurement procedure using MassChrom2D to combine chromatography MALDI data for determining the copolymer composition. Modified from Ref. [101].

Huijser et al. developed a software to establish the composition and topology of different linear chains of poly(lactide-co-glycolide) (PLLGA) which is used in drug delivery implants due to its advantageous properties as biodegradable and biocompatible materials [102]. The contour maps generated from the MALDI-MS analysis by using an in house developed software. The results show a significant difference in average composition between random and block copolymer chains. In the case of PLLGA, the line with a constant slope crossing the average composition do not pass through the origin, underlining a block-like structure, in contrast to PDLLGA which reveals a random like copolymer (Figure 8). Such analytical results are very useful for synthetic chemists, as they indicate an influence in the incorporation rate of a monomer between D-lactide and L-lactide. As it is shown in this study, 1-D MS can also have potential for the detailed analysis of polymer architectures with the help of a powerful data interpretation software. 

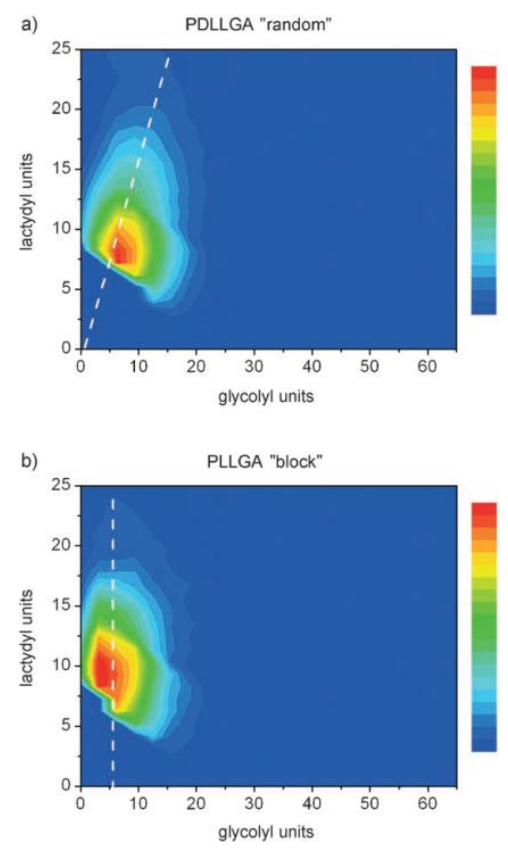

833 Figure 8. Contour plots of a) PDLLGA and b) PLLGA for cyclic structures plotted with 834 lactydyl units. Reproduced from Ref. [102].

In a contribution by Vivo-Truyols et al., a PS- $b$-PI block copolymer was analyzed by SEC and, subsequently, MALDI-ToF MS [103]. The overall architecture was then determined from the combined analysis of each fractions. This approach can only be used for non-overlapping isotopic patterns, otherwise regression is used to deconvolute the overlap. The limitation of this method is that the whole spectrum cannot be fitted, and regression can only be applied to

841 the center, which is not representative for the total polymer composition. Complications in 842 both mass resolution and processing power of the computer have also to be taken into 843 account. Nonetheless, this method can produce high-throughput data, offering a fast analysis 844 and yielding kinetic information on copolymers - satisfying both analysts and synthetic 845 chemists. In a related study, Willemse et al. portrayed the analysis and microstructure of PS$846 b$-PI block copolymers [104]. The authors showed that MALDI-ToF MS can provide 847 information on parameters such as chain length, composition, block properties or 848 microstructures. It was also shown that overlapping peaks in a copolymer distribution can be 
interpreted by using NMR as a feedback method if the integrals do not overlap excessively, and thus are not affecting the evaluation of the average composition. In addition, contour plots

851 were produced revealing the number of monomer units of each monomer and their microstructures. Wilczek-Vera et al. analyzed PS- $b$ - $\alpha m$ PS by MALDI-MS and ${ }^{1} \mathrm{H}$ NMR spectroscopy, where both techniques were required for determination of the block lengths and chemical composition [105]. The Schulz-Zimm model correlates both experimental and theoretical distributions, and can also be applied to triblock copolymers. The model is a rapid method to obtain a complete composition of the copolymer. It was pointed out that anionic ring-opening polymerization (AROP) produces polymers with narrow Đ values. However, it should be noted that the narrowness does not mean that the copolymer is structurally less complex.

Block copolymers are very important in polymer science and have found many applications in diverse fields. Different block copolymer materials have successfully characterized by utilizing various analytical techniques (thermal techniques or LC methods) in combination with MS. Additionally, the urge to have more automated systems to produce data in a faster manner and understand block copolymer structures and architectures, has brought advanced informatics into polymer science.

\subsection{Statistical copolymers}

Statistical copolymers are composed of monomers, which form a sequence according to a statistical rule. Many copolymers form statistical sequences, such as poly(2-phenyl-2oxazoline)-stat-(2(dec-9-enyl-2-oxazoline)). These are cationic polymers and due to their importance in biology and medicine, different analytical methods have been utilized in combination for their detailed characterization. The first example where statistical polymers 
were studied using a 2D setup was reported by the Schoemakers group, who analyzed block and statistical copolymers via gradient elution liquid chromatography (GELC), Pyrolysis-GC MS, SEC and capillary electrophoresis-ultraviolet (CE-UV) [106]. All four of these separation techniques provided different complementary information, none of which were conclusive on their own, thus, showing that hyphenated techniques can be essential to characterize complex statistical copolymers. First, Py-GC-MS was used to determine the average chemical composition. Subsequently, GELC was applied to further study the chemical-composition distribution. Offline GELC of these statistical copolymers showed two separated fractions, which were both bimodal in nature. An addition of a SEC dimension revealed that separation of these fractions in GELC dimension was not based on differences in the molar mass (Figure 9). This was rationalized by the presence of an ionic fraction in the samples of statistical copolymers, resulting from either chain-transfer reactions or termination by addition of water. Confirmation of this rationale was obtained by further analysis with $\mathrm{CE}$ and the results revealed that cationically charged polymers were predominant with only a minority being neutral. All of these analyses show that the statistical copolymers are not monomodal in comparison to block copolymers, but had different end groups and probably a different chemical composition per polymer chain0.

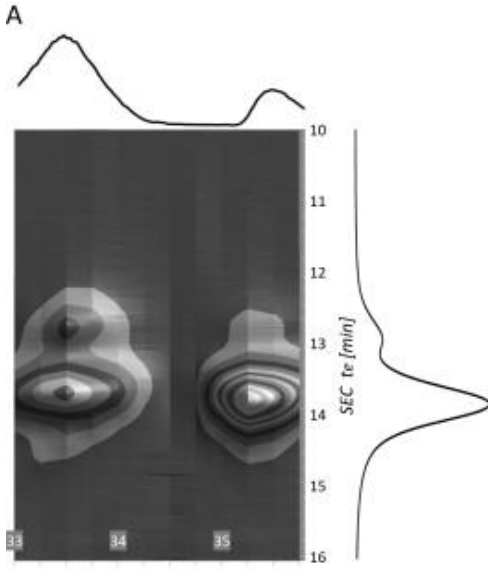

GELC th [min]

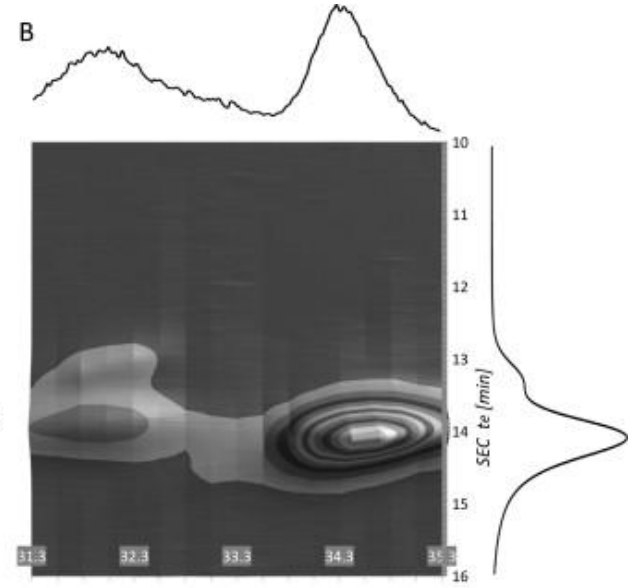

GELC tR [min] 
893 Figure 9. Off-line comprehensive 2D (GELC)/ $\times /$ SEC chromatograms of (polyPhOx ${ }_{20}-$ stat-

$\left.894 \mathrm{DecEnOx}_{40}\right)(\mathrm{A})$ and polyPhOx $\mathrm{x}_{40}-$ stat-DecEnOx 20 (B). Reproduced from Ref. [106].

895

896

897

898

899

900

901

902

903

904

905

906

907

908

909

910

911

912

913

914

915

916

917

\subsection{Random copolymers}

Random copolymers are quite challenging to analyze - in particular when aiming for a sequence determination [81]. An example of MS/MS analysis of random copolymers was recently reported which involved MALDI-ToF-MS/MS on samples containing styrene and dimethylsilylstyrene (DMSS) repeating units [107]. MS/MS provided detailed structural information and sequence coverage by revealing comonomer localization along the copolymer chains. A polystyrene oligomer end-capped by a block DMSS was easily differentiated from the random samples by isolating and fragmenting individual lithiated oligomers. From the MS/MS data, it was found that the localization of the DMSS block differs depending on whether $p$-DMSS or $m$-DMSS is used during the synthesis. Sequence information could be obtained by following the mass shifts occurring on the different fragment series containing either the terminating or the initiating end of the precursor oligomer ion that was being fragmented.

Huijser et al. used MALDI-ToF MS spectra to determine the reactivity ratios of the employed monomers, even with a long chain length model, for random, gradient, alternating and block-like copolymers [108]. This study represents a way to establish kinetic analysis by simply acquiring mass spectra. It was shown that, if analyzed and used properly, a MALDIMS method can reveal many details such as: reactivity ratios, composition, length of all chains and microstructures. Additionally, ESI-MS also shows a great promise for characterization of such complex copolymer mixtures with the addition of an IM dimension. Different blends of homopolymers and random copolymers have been studied by ESI-IM-MS [109]. Even from samples containing relatively low molar mass analytes, ESI produces ions in 
many different charge states readily, making the spectra harder to analyze without the IM dimension. Furthermore, folding transitions of the polymer chain can also be detected in the IM-MS spectra of mobility separated charge states. One aspect to highlight is the investigation of random copolymers PEO-r-PPG and PEO-r-PPO and their blends. This demonstrates that ESI-IM-MS can be a cost effective analytical method where the component in a complex mixture can be separated and investigated according to their architectural differences. In addition, Engler et al. used copolymer composition numbering tool (COCONUT), a computational method to establish the compositional distribution from MS spectra and the microstructures of random/block copolymers of PS-co-PI via AROP [110]. Isobaric species and overlapping isotopes could also be resolved using a method based on linear programming. Nonetheless, this method is only semi-quantitative, which is a remaining challenge in polymer science and mass spectrometry.

Overall, different hyphenated analytical techniques, combined with computer-based data processing methods, provides an effective tool to determine the lengths of the blocks and distinguishing between different architectures and sequences of block, random or statistical copolymer chains. The goal of these techniques and hyphenations is to aid chemists in tuning their polymers for their industrial and/or biological applications, as well as understanding and optimizing their chemical reactions.

\section{Third dimension of complexity: complex polymers}

With increasing demand on advanced polymer architectures for diverse applications, polymers are becoming more and more complex in their architecture, and at the same time, more challenging to characterize. Star-shaped, graft 'like' and branched polymers in particular are in the focus of current research efforts. Even these non-linear architectures are analyzed by many of the established methods presented above. 


\subsection{Graft 'like' polymers}

So far, only a few graft copolymers have jointly been investigated by MS and hyphenated techniques. One such example was PEO-g-poly(vinyl alcohol) (PEO-g-PVAl), which was used for instant-release tablet coatings due to its mechanical properties [111]. This particular graft copolymer was analyzed by 2D $($ LACCC $\times$ SEC) experiments, which showed that no free PEO was left after the radical polymerization. In addition, MALDI-ToF MS experiments were performed and confirmed this finding. Both complementary methods yielded the same basic results and furthermore, 2D-LC allowed the quantification of the degree of grafting. Comb-like polymers are of wide interest due to their unique architecture, however, they have not been analyzed widely either by MS or liquid chromatography. As an example, Adler et al. showed that such copolymers based on hydrophilic PEO and PMAA units, could be characterized via hyphenated techniques to MS [112]. Firstly, the LACCC conditions for PEOs were established in order to identify the presence of possible different end groups, followed by SEC for the molar mass measurement of PEO-comb-PMAA. The presence of the PEO macromonomer was confirmed by this method, as complemented by FT-IR. To conclude this analysis of hydrophilic copolymers, the authors showed that hyphenation and complementary methods are of necessity to evaluate their architecture of polymers, thereby quantifying the degree of grafting and, in addition, determining the monomer feed.

Graft 'like' copolymers have, to the best of our knowledge, hardly been analyzed via MS, nonetheless, $2 \mathrm{D}$ chromatography has been performed fairly often. In this respect, the 2D-LC based technique is very powerful for polymer analysis, especially with LACCC for one of the employed monomers. Moreover, the offline analysis by MALDI-ToF MS allows an additional dimension and, thus, provides further characteristics of the observed architectures. Both 2DLC and MS as hyphenated methods are versatile tools for the architecture characterization of 
graft 'like' polymers. The major drawbacks of a 2D chromatographic system are its expense, time-requirement, and the lack of appropriate standards, leading to erroneous results.

\subsection{Cyclic polymers}

Cyclic polymers have very different physical properties when compared to their linear homologues and are applied in a wide range of fields, for example, commercial polycarbonates and polyesters for the perfume industry. In contrast to linear species, no chain ends are present as a result from ring closure. Many groups have studied the difference between linear and cyclic polymers using several different ionization methods, hyphenations and complementary methods to obtain as much knowledge and architectural information as possible. For example, Yol et al. have compared both linear and cyclic homopolymers of PS and poly(butadiene) (PB), which showed significant differences in their MALDI MS/MS spectra [113]. In particular, in the case of linear polymers, a noticeable "depolymerization" was observed, and low mass radical ions were predominant; in comparison, cyclic polymers of similar molar masses gave fragments of higher molar mass with higher relative abundance. In either case, MALDI-ToF MS/MS can determine the end group (for linear polymers) or the linker (for cyclic polymers) used, and is applicable to polymers prepared via different polymerization techniques. Maslinska-Solich et al., was able to differentiate linear and macrocyclic oxazolidine-based polymers just by analyzing their MS spectra, and assigning specific peaks to polymer chains with and without end groups [114,115]. Wachsen et al., used three complementary methods to compare linear and cyclic polymers [116]. PLA was used due to its importance as a synthetic polymer, since it is extensively used in biocompatible and biodegradable materials. SEC, LACCC, and MALDI-MS were used to perform a detailed characterization of PLAs. These complementary methods were essential to either determine the structure or separate the different architectures formed during various polymerization 
techniques. LACCC is a very beneficial technique, however, it is very time consuming due to the many parameters that need to be optimized. Nevertheless, it allows the differentiation between the components with different end groups independently from their molar mass. Osaka et al. studied PLA by ESI- and MALDI-MS where differences between linear and cyclic architectures becomes apparent [37]. Different fragment ion series were observed in the ESI-MS/MS spectra: three series for the linear and one for the cyclic polymers. Compared to ESI-MS/MS results, MALDI-MS/MS spectra showed the same fragment series for cyclic architectures but differed for linear architectures (only one fragment series was formed). A less common technique was used by McDonnell et al. for the same purpose: sustained off resonance irradiation (SORI)-CAD-FT-ion cyclotron resonance (ICR). In contrast to the previous approaches, this method has very high mass resolution [117]. This allowed to carry out high accuracy mass measurements of the fragment ions which essentially helped to derive different fragmentation mechanisms observed for both linear and cyclic architectures.

Although MS techniques are widely used for the analysis of linear and cyclic polymers, quantification of cyclic and linear chains in an analyte mixture has hardly been addressed by using a hyphenated method. As an exception, Wang et al. applied surface layer (SL)-MALDIToF MS to quantify and determine different architectures (linear and cyclic) in PS films that were spin cast from blends [118]. Since the linear and cyclic polymers in the blend differ in mass, no MS/MS was required, but its use with either ESI- or MALDI-MS would make it possible to differentiate architectures and/or sequences that have the same mass. MALDI- and ESI-MS can cause chemical changes to labile polymers, leading to erroneous data when using an inappropriate solvent, salt or matrix is used. Such changes can be detected using IM-MS where charge states and/or overlapping components with different architecture can be deconvoluted [119]. 
In conclusion, 2D chromatography methods, MS, MS/MS and/or IM-MS have been used to characterize cyclic synthetic (co)polymers. Direct ESI- and MALDI-MS can be enough to differentiate between linear and cyclic oligomers, if there is high enough resolution. However, this is difficult to achieve for high molar masses. CAD is also used for this specific differentiation, however, the poor fragmentation efficiency at higher $\mathrm{m} / \mathrm{z}$ values, typically limits its application to lower molecular weight polymers. IM-MS, on the contrary, is a much faster technique that can be used to differentiate linear and cyclic polymers.

\subsection{Star-shaped polymers}

Star-shaped polymers are synthesized via different techniques (for example "core-first", "arm-first" or "graft-onto" methodologies) and are attractive materials for diverse applications, such as drug delivery or material science. However, their characterization is not facile due to their chemical structure. Several analytical techniques have been used to establish a full characterization of star-shaped polymers.

The RAFT polymerization technique is one of the commonly used methods for the preparation of star-shaped polymers [61,120,121]. Star-shaped polymers of paraacetoxystyrene and its by-products obtained during RAFT polymerization was analyzed by Chaffey-Millar et al. with MS techniques [120]. In this example, ESI-MS proved to be a powerful tool to differentiate between "star-star couples", terminated with initiator fragments and linear polymers. In addition to ESI-MS analysis, a kinetic model for the polymerization conclude that complementary methods are vital to explain experimental observations. 
mass. PB-based star-shaped polymers were synthesized by Allgaier et al. and the products were analyzed with MALDI-ToF MS [122]. The investigation was limited to low molar masses, however, it was still sufficient to confirm the star-shaped architecture of the polymers. The results were extrapolated to high molar masses through the use of a theoretical modeling. A four-arm PEO-based star-shaped polymer was obtained from AROP and subsequently transformed into an eight-shaped polymer via end group modification and twofold intramolecular ring closure [123]. The final products as well as all intermediates are unambiguously identified by MALDI-MS. IM-MS was also used to examine the conformations of linear PCLs and star shaped PCLs having different topologies [124]. By using theoretical molecular dynamics calculations, two major conformations were found for such polymers (near-spherical and elongated) depending on the charge state and the length of the polymer. Furthermore, these findings were supported by CAD experiments. In light of the results that were obtained by both experiments and simulations, it was concluded that the final conformations depend on the degree of polymerization, charge state, and the branching ratio of the polymer.

Star-shaped block copolymers can also be prepared through the coupling to a core, and are of great interest for drug delivery applications. Li et al. showed that characterization of such star-shaped polymers is difficult [125]. Star-shaped PCL- $b$-PEGs were characterized via SEC and MALDI-ToF MS, where MALDI analyses provided information on both the molar masses and the structural identity. The SEC analyses provided additional confirmation and complementary information on the molar masses. One has to be cautious when a star is composed of two different monomers due to differences in the ionization behavior. In this case, a study with a 2D (LACCC $\times$ SEC) system would be appealing, taking the critical conditions of both homopolymers (PEG and PCL) into account to ensure that the star is not missing an arm. Furthermore, Rudolph et al. showed that MALDI, static light scattering 
(SLS) and 2D-LC are essential to obtain absolute molar masses, and to confirm the architecture of their star-shaped $\left[\mathrm{PEO}_{28}-b-\mathrm{PEtOx}\right]_{8}$, which was synthesized by the coppercatalyzed azide-alkyne cycloaddition reaction (CuAAC). These techniques are necessary considering that NMR spectroscopy and SEC are not satisfactory to fully characterize these systems [126].

Another type of architecture, branched-based star polymers, was investigated by $\mathrm{Yu}$ et al. by MALDI-MS, even at high molar masses [127]. The monomer employed here was EO, which - due to its neutral properties and high ionization efficiency - enables an investigation of polymers even at high molar masses and non-linear architectures. Here, MALDI-MS proves to be a powerful tool to identify stars with different numbers of arms.

Despite the successful characterizations of star-shaped polymer systems in the above examples, analysis of such materials still remains complex in nature. From the diverse examples portrayed, it is clear that star-shaped polymers and their possible side products e.g. star-star couples, are distinguishable by MS only. However, IM-MS and 2D-LC can provide additional information to differentiate topologies, and moreover this can be supported by MS/MS or other complementary techniques such as LS or small angle X-ray scattering (SAXS), to determine branching ratios, charge states and the degree of polymerization. The analytical potential of IM-MS is increasingly being appreciated and its speed in analysis is being remarkably acknowledged. Although some methods are still more suitable for high molar masses e.g. LS, asymmetric flow field-flow fractionation (AF4) and analytical ultracentrifuge AUC. It is envisaged that complementary techniques such as IM-MS, 2D-LC, LS, AF4 should further support the characterization of these polymer systems. 


\subsection{Branched polymers}

The analysis of dendritic materials with MALDI-ToF MS has been carried out utilizing different matrices, doping agents, solvents, delayed extractions and polymer concentrations. Many examples based on different types of polymers and different numbers of dendritic generations as well as branched or hyperbranched polymers have been characterized via direct MALDI MS and also by a range of hyphenated techniques.

\subsubsection{Amidoamines and imines}

Numerous methods are used to analyze and evaluate different architectures of such compounds. FT-ICR MS was utilized to characterize poly(amidoamine) (PAMAM) based dendrimers - their trade name is Starburst - which exhibit good biocompatibility [128]. Highresolution spectra can be used to determine the $Đ$ values of dendrimers, at least for those of low molar masses. Moreover, this specific instrumentation offers resolving power for high molar masses even of rather disperse samples. MS/MS is useful in this particular case to determine the faults introduced in the synthetic route and to identify the detailed architecture by the specific fragmentation reactions of defect and non-defect dendrimers. This can be done using fragments resulting from competitive retro-Michael additions on the PAMAM: from multiply charged dendrimer ions, two major types of fragments are formed during MS/MS experiments due to the separation of charges [129]. Amide-based dendrimers have been used widely in studies designed to fully understand the ECD mechanism [130]. Unexpected results were discovered such as $\mathrm{b} / \mathrm{y}$ cleavages (cleavage after $\mathrm{C}=\mathrm{O}$ towards the outer layer), $\mathrm{S}, \mathrm{E}$ dissociations (direction of the cleavage start, end respectively) and minor c, $\mathrm{z}$ (cleavage after $\mathrm{N}-\mathrm{H}$ towards the outer layer) fragmentations. The fragmentation patterns were compared to poly(propylene imine) (PPI) dendrimers, which lack the amide groups. The comparison showed that macromolecular properties, intramolecular charge-solvation and energy barriers 
are important determinants for the fragmentation pathways observed, and thus, for the architecture deduced from them. In addition, IM-MS has been used to separate dendrimer constituents and turned out to be much faster than LC or capillary zone electrophoresis (CZE) [131]. Unique cross-sections would be assigned to 'perfect and 'imperfect' dendrimers, thus unveiling their architectures and complementing the MS/MS data. Furthermore, the results correlated with NMR spectroscopy and SAXS, thus emphasizing the versatility of this technique. Another application of IM-MS in combination with molecular modeling on PAMAM dendrimers concerns the dependence of conformation on the degree of protonation. The results obtained by theoretical calculations show that changes in the conformation are dictated by electrostatic repulsion, which is in agreement with the experimental IM-MS results. On the basis of these findings, IM-MS supported by computational chemistry is a promising tool for future studies on the architectures of higher generation dendrimers [132]. PAMAM dendrimers of higher molar mass were also studied by MALDI-MS and ISD [133]. Different matrices were found to result in different fragmentation patterns. The fragments obtained from ISD of PAMAM dendrimers correlate well with those from ECD of such materials.

Dendrimers of the PPI-type have been studied using different fragmentation techniques to elucidate their architectures. For example, Adhiya et al. examined the conformation of such dendrimers in solution and the gas phase by ESI-CAD and MALDI-PSD-MS [134]. Solutions in different solvents were used and diverse fragmentation pathways, such as the cleavage of outer branches from singly protonated ions, were monitored as a function of the solvent (polar/protic vs. non-polar). Lower intensities were observed with non-polar solvents, indicating differences in the dendrimer architectures and the conformations probed. Meijer and co-workers have reviewed the same type of dendrimers in detail by using ESI-CAD, and similar pathways were observed [135]. Further, low energy ESI-MS has been applied to 
monitor the growth, fragmentations and shape of such dendrimers [136,137]. In addition, HPLC was hyphenated to ESI-MS and used to detect defects in the dendrimers, however, this represents a demanding task due to co-elution of components and isomerism of structures. Adding MS/MS experiments should at least partly resolve these problems by providing information on the different architectures eluting [138]. Branched PEI was investigated under ESI- and MALDI-MS conditions with CAD. These polymers were widely studied due to their reputation as a "golden standard" for gene delivery applications [49]. Three low abundant fragmentation series are detected, arising from a C-N bond cleavage. Their relative abundances provide useful insight on the branching architectural features of the examined PEI samples.

\subsubsection{Ethers and esters}

Ether based dendrimers have several application domains and MALDI-ToF MS is the traditionally used method for their characterization. MALDI-MS has been utilized to determine the structure and end groups of hyperbranched systems and, thus, differentiate cyclic structures of 3-[2-[2-(2-hydroxyethoxy)ethoxymethyl)-3'-methyl-oxetane (PHEMO), which is used as polymer electrolyte for reinforcement of polyelectrolyte membranes [139]. FT-ICR combined with liquid secondary ion mass spectrometry (SIMS) was used to evaluate, the fragmentation behavior of poly(ether)-type dendrimers with lateral terpyridine moieties and their complexes with iron(II) ions, for an architectural investigation [140]. Hyperbranched 3-ethyl-3-hydroxymethyloxetane trimethylolpropaneoxetane (TMPO)-based polyethers of low molar masses were investigated with ToF-SIMS [141]. The use of this technique with dendrimers of early generations allowed the determination of the degree of branching. However, high molar masses are not accessible with this method. 

homogeneity, disperse nature and the high laser power requirement for their ionization made the analysis of the spectra of such dendrimers intricate [142]. Fragmentations are induced insource due to the high laser intensity, indirectly affirming the architecture by the presence of a series of cleaved off branch units. In a different study, MALDI-MS was applied to verify the synthetic route to such dendrimers [143]. The findings pointed out that traditional solutionbased methods, such as NMR spectroscopy and SEC, are not powerful enough, and that hyphenated techniques and/or direct MS is required. Polyesteramides, as biodegradable polymers, were studied with MALDI- and ESI-MS giving different results, such as $\mathrm{OH}$ groups being present in the ESI spectra but not in the MALDI spectra [144]. This study demonstrated the need for using more than one ionization technique in order to determine architecture through direct MS. The other observation was that ISD is observed with MALDI and not with ESI; thus the analysis of the MALDI data should be carried out carefully due to fragments that lead to erroneous architecture deduction. Finally, Koster et al. used both ECD and low energy CAD to analyze hyperbranched oligomers and concluded that ECD produces more fragments, because it deposits higher internal energies to cause consecutive 1180 fragmentations; however, ECD did not produce complementary sequence information, because the new fragments were internal [145]. Thus one has to be aware, when using the data of different fragmentation techniques, that the fragment production mechanisms permit the derivation of the correct architecture.

Hyperbranched polyacrylates, in which the branches grow out of the ester groups, have been prepared by ATRP of acrylate inimers (an inimer can react as an initiator or monomer) [26]. Their architectures were analyzed by MS/MS. Fragmentation occurs by sigmatropic 1,5$\mathrm{H}$ rearrangements over the ester groups, but not by rearrangement of $\mathrm{H}$ atoms in the polyacrylate chain. The rearrangements over the side chain provide information about the 
branching architecture. The fragments produced reveal branching sites and branch sizes, and are completely different from fragments generated by the isomeric linear architecture.

\subsubsection{Aromatic ring based}

Aromatic monomers with a variety of structures have been polymerized to form dendrimers. Such dendrimers, or branched systems, were analyzed via MS techniques (as well as other methods). For example, the Fréchet group applied MALDI-MS to study the functionalities at the surface and the focal point of dendritic aromatic poly(ether)s [146,147]. This method allowed monitoring of the purity and the growth unlike other standard polymer characterization methods. Aromatic poly(ether)s and block-like dendrimers with potential biological applications were characterized by MALDI-ToF MS, which enabled confirmation of the architecture generated using different monomers [148]. In this study, the choice of matrix and the sample preparation method were critical for obtaining informative mass spectra. Aromatic polyester dendrimers were analyzed by MALDI and laser desorption ionization (LDI) in the presence of different metal salts, which enabled the polymer to be ionized by cation attachment, so that the different generations formed could be identified and verified [149]. In a more recent study, branched and linear poly(aramide)s were compared by MALDI-IM-MS. Synthesized poly(aramide)s were compared to commercial Kevlar ${ }^{\circledR}$ and was found that in $\operatorname{Kevlar}^{\circledR}$ both linear and branched species are present [150]. Furthermore, IM separation enabled the distinction between branched meta-para aramide and para aramide $\left(\right.$ Kevlar $\left.^{\circledR}\right)$. In addition, CAD of both branched and linear products was performed and distinct fragments appeared from each sample, thus enabling differentiation between different architectures. This example demonstrates that how a fast and facile these techniques can be to control the production and quality of the material and how polymer characterization can improve in the future. A highly branched PS was synthesized by linking polystyrene anions 

architecture.

with 4-chlorodimethyl-silylstyrene and analyzed by MALDI-MS and HPLC [151]. Reverse phase temperature gradient interaction chromatography (RP-TGIC) was used first to fractionate the polymer according to the number of PS branches. The fractions were then characterized by MALDI-ToF MS. Resolution was higher with a RP column in comparison to a normal phase (NP) one, and increased with the number of branches. LACCC resulted in lower resolution than RP-TGIC. Non-aromatic branched polymers were investigated by Liu et al. who analyzed the compositions, sequences and architectures of small oligomers of a hyperbranched glycopolymer, prepared by atom transfer radical copolymerization of an acrylate monomer (A) and an acrylate inimer (B), both carrying mannose ester pendants [152]. MALDI-MS and/or ESI-MS data confirmed the incorporation of multiple inimer repeat units, which lead to formation of a hyperbranched material. $\mathrm{MS}^{\mathrm{n}}$ studies elucidated the atomic connectivity in various n-mers and specific sequences were distinguished from isomers based on the characteristic elimination of a bromomethane molecule. This provided definitive information about the comonomer connectivity in the copolymeric $\mathrm{AB}_{2}$ trimer and $\mathrm{A}_{2} \mathrm{~B}_{2}$ tetramer isomers. Consistent with the MS/MS data, IM-MS studies confirmed that only one of the possible $A_{2} B_{2}$ structures was formed (Figure 10). A comparison of the experimentally determined collision cross-section (CCS) of the detected isomer with the theoretical predictions for the two sequences conclusively showed that $\mathrm{BBA}_{2}$ is the prevalent tetrameric 


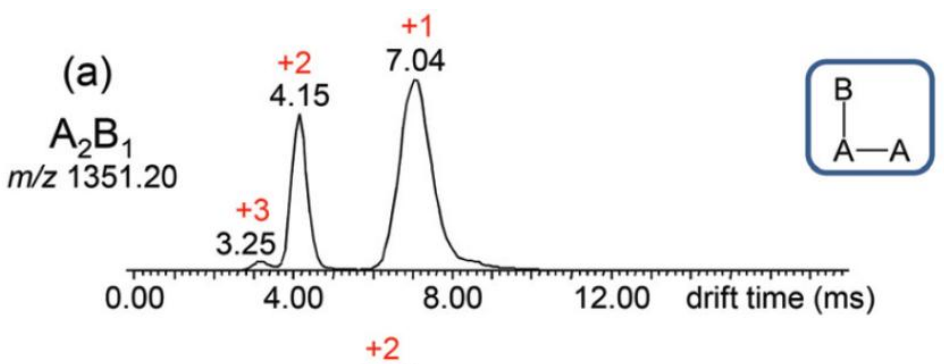

(b)

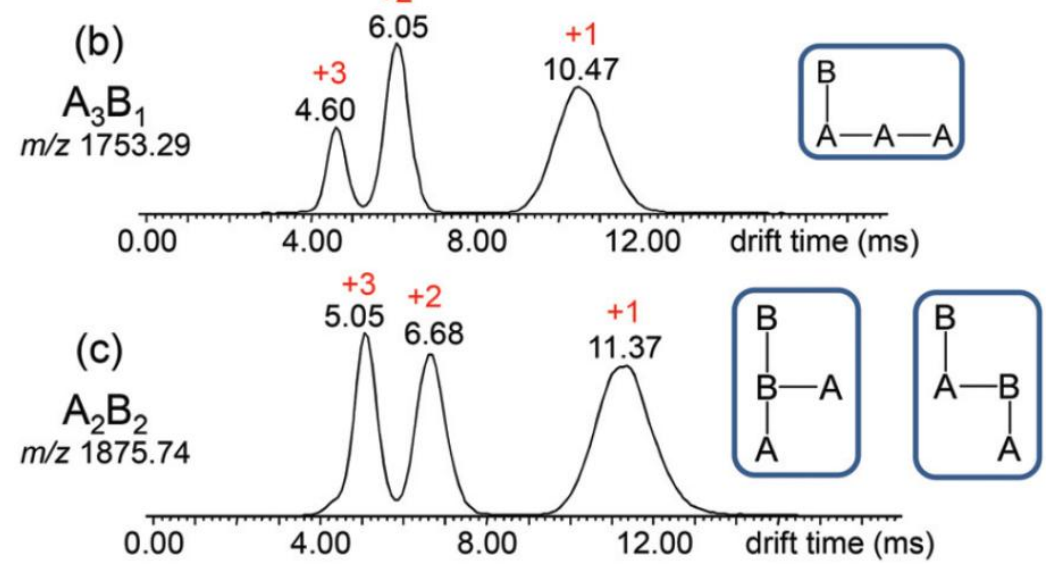

1235 Figure 10. (a - c) ESI-IM-MS drift time distributions of sodiated, $[\mathrm{M}+\mathrm{nNa}]^{\mathrm{n}+}$, glycopolymer 1236 samples having different comonomeric ratios. Three peaks are observed for all three samples, 1237 corresponding to ions with +1 to +3 sodium charges. The $A_{2} B_{1}$ trimer and $A_{3} B_{1}$ tetramer can 1238 only have the sequence shown, as they contain only one inimer unit. Based on MS/MS, the $1239 \mathrm{~A}_{2} \mathrm{~B}_{2}$ tetramer loses $\mathrm{CH}_{3} \mathrm{Br}$, which is compatible with the two sequences shown. Only the 1240 first, $\mathrm{BBA}_{2}$, has a simulated collision cross-section that matches the measured one. 1241 Reproduced from Ref. [152].

MALDI-ToF MS is extremely beneficial for characterizing branched polymers. By 1244 utilizing a higher laser power and fragmentation in MALDI-MS are suitable for samples with 1245 high branching and the determination of branching points. Therefore, various ionization 1246 techniques with different fragmentation methods should be used to support hypotheses. In 1247 some of the cases, CAD, ECD or ISD were used to establish possible defects in architectures. 1248 Specific cleavages enable the verification of the synthetic routes e.g. outer layer cleavages of 1249 specific bonds. Therefore, tandem mass spectrometry remains an important technique in 
characterizing the structural features in branched polymers. Furthermore, addition of IM dimension and molecular modeling to the analysis are useful for confirming their respective results regarding conformation changes and for providing complementary information on architectural differences.

\section{Conclusion}

The field of polymer science includes a very broad range of macromolecular species, with diverse structural complexity and molar masses. In the past 20 years, many applications involved synthetic polymers, which experience growing interest in the fields of biology and medicine. Many properties of synthetic polymers are important such as heterogeneity, topology, composition, functionality and/or molar mass. ESI-MS and MALDI-MS have been extensively used in the last two decades and have provided a great magnitude of information on the above-mentioned properties. Numerous developments have arisen recently in MS concerning high-resolution mass analyzers and higher sensitivity, which will boost MS applications in polymer science. Hyphenation of other analytical techniques to MS, for example LC, facilitates the determination of chemical heterogeneity and molar mass, and thus, enhances MS analysis. Furthermore, IM-MS is found to be of potential equivalence to LC, by separating molecules in the gas-phase relatively quickly according to their architectural differences without the need of excessive amounts of solvent consumption. In the future, further improvement and application of all these potential tools will help to investigate more complex architectures of different polymeric materials, which is still a challenging topic in terms of characterization. Moreover, automated software are being developed and continue to be implemented to support complicated data interpretation. MS remains to be a versatile technique and has a promising future in combination with other analytical and computational 
1274 methods for an improved analysis, and a better understanding of polymer systems with 1275 different architectures.

1276

\section{Acknowledgement}

1278 Financial support from the Thüringer Ministerium für Bildung, Wissenschaft und Kultur 1279 (grants no. B515-07008 and B715-08011) and from the National Science Foundations (CHE1280 1308307) is gratefully acknowledged. S. Crotty thanks Dr. A. Winter and Dr. K. Knop for 1281 discussions and corrections of this work.

\section{References}

[1] K. Kataoka, A. Harada, Y. Nagasaki, Block copolymer micelles for drug delivery: design, characterization and biological significance, Adv. Drug Deliv. Rev. 47 (2001) 113131.

1287

[2] A.P. Gies, Mass Spectrometry in Polymer Chemistry, Wiley, Weinheim, 2011, p. 33 128856.

1289 [3] V. Scionti, C. Wesdemiotis, Mass Spectrometry in Polymer Chemistry, Wiley, 1290 Weinheim, 2011, p. 57-84.

1291 [4] J.B. Fenn, Electrospray wings for molecular elephants Angew. Chem. Int. Ed. 42 1292 (2003) 3871-3894.

[5] M. Karas, F. Hillenkamp, Laser desorption ionization of proteins with molecular 1294 masses exceeding 10,000 daltons, Anal. Chem. 60 (1988) 2299-2301.

1295 [6] D.I. Carroll, I. Dzidic, R.N. Stillwell, K.D. Haegele, E.C. Horning, Atmospheric 1296 pressure ionization mass spectrometry. Corona discharge ion source for use in a liquid 1297 chromatograph-mass spectrometer-computer analytical system, Anal. Chem. 47 (1975) 2369$1298 \quad 2373$. 

polymer analyses up to $\mathrm{m} / \mathrm{z} 100000$ by laser ionization time-of-flight mass spectrometry, Rapid Commun. Mass Spectrom. 2 (1988) 151-153.

J. Falkenhagen, S. Weidner, Mass Spectrometry in Polymer Chemistry, Wiley, Weinheim, 2011, p. 209-235.

[9] M. Dole, L.L. Mack, R.L. Hines, R.C. Mobley, L.D. Ferguson, M.B. Alice, Molecular beams of macroions, J. Chem. Phys. 49 (1968) 2240-2249.

[10] G.E. Kassalainen, S.K. Ratanathanawongs Williams, Coupling thermal field-flow fractionation with matrix-assisted laser desorption/ionization time-of-flight mass spectrometry for the analysis of synthetic polymers, Anal. Chem. 75 (2003) 1887-1894.

[11] X. Li, Y.-T. Chan, G.R. Newkome, C. Wesdemiotis, Gradient tandem mass spectrometry interfaced with ion mobility separation for the characterization of supramolecular architectures, Anal. Chem. 83 (2011) 1284-1290. Weinheim, 2011, p. 281-318.

[13] P. Rizzarelli, S. Carroccio, C. Puglisi, Mass Spectrometry in Polymer Chemistry, Wiley, Weinheim, 2011, p. 437-465.

[14] N. Zhang, Y. Zhou, C. Zhen, Y. Li, C. Xiong, J. Wang, H. Li, Z. Nie, Structural characterization of synthetic polymers using thermal-assisted atmospheric pressure glow discharge mass spectrometry, Analyst 137 (2012) 5051-5056. thermal degradation of poly(propylene oxide) by electrospray and matrix-assisted laser desorption ionization, Polymer 36 (1995) 4927-4933. 
[16] C.J. Tsai, L.H. Perng, Y.C. Ling, A study of thermal degradation of poly(aryl-etherether-ketone) using stepwise pyrolysis/gas chromatography/mass spectrometry, Rapid Commun. Mass Spectrom. 11 (1997) 1987-1995.

[17] R. Lu, Y. Kamiya, Y.-Y. Wan, T. Honda, T. Miyakoshi, Synthesis of Rhus succedanea lacquer film and analysis by pyrolysis-gas chromatography/mass spectrometry, J. Anal. Appl. Pyrolysis 78 (2007) 117-124.

[18] S.L. Madorsky, S. Straus, High vacuum pyrolytic fractionation of polystyrene, Ind. Eng. Chem. 40 (1948) 848-852.

[19] L.A. Wall, Mass spectrometric investigation of the thermal decomposition of polymers, J. Res. Natl. Bur. Stand. (U.S.) 41 (1948) 315-322.

[20] C. Wesdemiotis, N. Solak, M.J. Polce, D.E. Dabney, K. Chaicharoen, B.C. Katzenmeyer, Fragmentation pathways of polymer ions, Mass Spectrom. Rev. 30 (2011) 523559.

[21] E. Caló, V.V. Khutoryanskiy, Biomedical applications of hydrogels: A review of patents and commercial products, Eur. Polym. J. 65 (2015) 252-267.

[22] T. Hanemann, D.V. Szabó, Polymer-nanoparticle composites: from synthesis to modern applications, Materials 3 (2010) 3468.

[23] E. Altuntaş, A. Krieg, A. Baumgaertel, A.C. Crecelius, U.S. Schubert, ESI, APCI, and MALDI tandem mass spectrometry of poly(methyl acrylate)s: A comparison study for the structural characterization of polymers synthesized via CRP techniques and the software application to analyze MS/MS data, J. Polym. Sci., Part A: Polym. Chem. 51 (2013) 15951605.

[24] O. Laine, S. Trimpin, H.J. Raeder, K. Muellen, Changes in post-source decay fragmentation behavior of poly(methyl methacrylate) polymers with increasing molecular 
weight studied by matrix-assisted laser desorption/ionization time-of-flight mass spectrometry, Eur. J. Mass Spectrom. 9 (2003) 195-201.

[25] C.D. Borman, A.T. Jackson, A. Bunn, A.L. Cutter, D.J. Irvine, Evidence for the low thermal stability of poly(methyl methacrylate) polymer produced by atom transfer radical polymerisation, Polymer 41 (2000) 6015-6020.

[26] K. Chaicharoen, M. Polce, A. Singh, C. Pugh, C. Wesdemiotis, Characterization of linear and branched polyacrylates by tandem mass spectrometry, Anal. Bioanal. Chem. 392 (2008) 595-607.

[27] M.-S. Giguère, P.M. Mayer, Climbing the internal energy ladder: the unimolecular decomposition of ionized poly(vinyl acetate), Int. J. Mass Spectrom. 231 (2004) 59-68.

[28] R. Giordanengo, S. Viel, B. Allard-Breton, A. Thévand, L. Charles, Positive mode electrospray tandem mass spectrometry of poly(methacrylic acid) oligomers, Rapid Commun. Mass Spectrom. 23 (2009) 1557-1562.

[29] J.J.L. Bryant, J.A. Semlyen, Cyclic polyesters: 6. Preparation and characterization of two series of cyclic oligomers from solution ring-chain reactions of poly(ethylene terephthalate), Polymer 38 (1997) 2475-2482.

[30] J.J.L. Bryant, J.A. Semlyen, Cyclic polyesters: 7. Preparation and characterization of cyclic oligomers from solution ring-chain reactions of poly(butylene terephthalate), Polymer 38 (1997) 4531-4537.

[31] P. Kilz, W. Radke, Application of two-dimensional chromatography to the characterization of macromolecules and biomacromolecules, Anal. Bioanal. Chem. 407 (2015) 193-215.

[32] K. Yamanaka, Y. Kimura, T. Aoki, T. Kudo, End-group analysis of bacterially produced poly(3-hydroxybutyrate): discovery of succinate as the polymerization starter, Macromolecules 42 (2009) 4038-4046. 
[33] P. Rizzarelli, Matrix-assisted laser desorption ionization time-of-flight/time-of-flight tandem mass spectra of biodegradable polybutylenesuccinate, Rapid Commun. Mass Spectrom. 27 (2013) 2213-2225.

O. Laine, T. Laitinen, P. Vainiotalo, Characterization of polyesters prepared from three different phthalic acid isomers by CID-ESI-FT-ICR and PSD-MALDI-TOF mass spectrometry, Anal. Chem. 74 (2002) 4250-4258.

[35] V. Scionti, C. Wesdemiotis, Electron transfer dissociation versus collisionally activated dissociation of cationized biodegradable polyesters, J. Mass Spectrom. 47 (2012) 1442-1449.

[36] B.C. Katzenmeyer, L.R. Cool, J.P. Williams, K. Craven, J.M. Brown, C. Wesdemiotis,

Electron transfer dissociation of sodium cationized polyesters: Reaction time effects and combination with collisional activation and ion mobility separation, Int. J. Mass Spectrom. 378 (2015) 303-311.

1384

I. Osaka, M. Watanabe, M. Takama, M. Murakami, R.

R. Arakawa, Characterization of linear and cyclic polylactic acids and their solvolysis products by electrospray ionization mass spectrometry, J. Mass Spectrom. 41 (2006) 1369-1377.

[38] W.J. De, O. Coulembier, P. Dubois, P. Gerbaux, Collision-induced dissociation of polymer ions: Charge driven decomposition for sodium-cationized polylactides and isomeric end-group distinction, Int. J. Mass Spectrom. 308 (2011) 11-17.

[39] P. Terrier, B. Desmazieres, J. Tortajada, W. Buchmann, APCI/APPI for synthetic 1391 polymer analysis, Mass Spectrom. Rev. 30 (2011) 854-874.

1392 [40] G. Hart-Smith, A review of electron-capture and electron-transfer dissociation tandem 1393 mass spectrometry in polymer chemistry, Anal. Chim. Acta 808 (2014) 44-55. 
[41] A. Baumgaertel, C. Weber, K. Knop, A. Crecelius, U.S. Schubert, Characterization of different poly(2-ethyl-2-oxazoline)s via matrix-assisted laser desorption/ionization time-offlight tandem mass spectrometry, Rapid Commun. Mass Spectrom. 23 (2009) 756-762.

[42] E. Altuntaş, K. Kempe, A. Crecelius, R. Hoogenboom, U.S. Schubert, ESI-MS \& MS/MS analysis of poly(2-oxazoline)s with different side groups, Macromol. Chem. Phys. 211 (2010) 2312-2322.

[43] E. Altuntas, C. Weber, K. Kempe, U.S. Schubert, Comparison of ESI, APCI and MALDI for the (tandem) mass analysis of poly(2-ethyl-2-oxazoline)s with various endgroups, Eur. Polym. J. 49 (2013) 2172-2185.

[44] E. Altuntas, C. Weber, U.S. Schubert, Detailed characterization of poly(2-ethyl-2oxazoline)s by energy variable collision-induced dissociation study, Rapid Commun. Mass Spectrom. 27 (2013) 1095-1100.

[45] K. Knop, R. Hoogenboom, D. Fischer, U.S. Schubert, Poly(ethylene glycol) in drug delivery: pros and cons as well as potential alternatives, Angew. Chem. Int. Ed. 49 (2010) 6288-6308.

[46] A.J.R. Lasprilla, G.A.R. Martinez, B.H. Lunelli, A.L. Jardini, R.M. Filho, Poly-lactic acid synthesis for application in biomedical devices - A review, Biotech. Adv. 30 (2012) 321-328.

[47] N. Adams, U.S. Schubert, Poly(2-oxazolines) in biological and biomedical application contexts, Adv. Drug Deliv. Rev. 59 (2007) 1504-1520.

[48] E. Altuntaş, K. Knop, L. Tauhardt, K. Kempe, A.C. Crecelius, M. Jäger, M.D. Hager, U.S. Schubert, Tandem mass spectrometry of poly(ethylene imine)s by electrospray ionization (ESI) and matrix-assisted laser desorption/ionization (MALDI), J. Mass Spectrom. 47 (2012) 105-114. 
[49] E. Rivera-Tirado, C. Wesdemiotis, Characterization of polyethylenimine by electrospray ionization and matrix-assisted laser desorption/ionization, J. Mass Spectrom. 46 (2011) 876-883.

[50] V. Mass, W. Schrepp, B. von Vacano, H. Pasch, Sequence analysis of an isocyanate oligomer by MALDI-TOF mass spectrometry using collision induced dissociation, Macromol. Chem. Phys. 210 (2009) 1957-1965.

[51] K.M. Wollyung, C. Wesdemiotis, A. Nagy, J.P. Kennedy, Synthesis and mass spectrometry characterization of centrally and terminally amine-functionalized polyisobutylenes, J. Polym. Sci., Part A: Polym. Chem. 43 (2005) 946-958.

[52] C. Schilli, M.G. Lanzendoerfer, A.H.E. Mueller, Benzyl and cumyl dithiocarbamates as chain transfer agents in the RAFT polymerization of $\mathrm{N}$-isopropylacrylamide. In situ FTNIR and MALDI-TOF MS investigation, Macromolecules 35 (2002) 6819-6827.

[53] A. Mahon, T.J. Kemp, J.E. Varney, P.J. Derrick, Ions derived from linear polysulfide

oligomers using matrix-assisted laser desorption/ionisation time-of-flight mass spectrometry, Polymer 39 (1998) 6213-6217.

1433 [54] S.T. Ellison, A.P. Gies, D.M. Hercules, S.L. Morgan, Py-GC/MS and

P. Rizzarelli, C. Puglisi, Structural characterization of synthetic poly(ester amide) from

sebacic acid and 4-amino-1-butanol by matrix-assisted laser desorption ionization time-of1438 flight/time-of-flight tandem mass spectrometry, Rapid Commun. Mass Spectrom. 22 (2008) $1439 \quad 739-754$.

[56] S. Trimpin, S.M. Weidner, J. Falkenhagen, C.N. McEwen, Fractionation and solventfree MALDI-MS analysis of polymers using liquid adsorption chromatography at critical 
conditions in combination with a multisample on-target homogenization/transfer sample preparation method, Anal. Chem. 79 (2007) 7565-7570.

[57] R. Peters, Y. Mengerink, S. Langereis, M. Frederix, H. Linssen, J. van Hest, S. van der Wal, Quantitation of functionality of poly(methyl methacrylate) by liquid chromatography under critical conditions followed by evaporative light-scattering detection. Comparison with NMR and titration, J. Chromatogr. A 949 (2002) 327-335.

[58] T. Gruendling, M. Guilhaus, C. Barner-Kowollik, Quantitative LC-MS of polymers: Determining accurate molecular weight distributions by combined size exclusion chromatography and electrospray mass spectrometry with maximum entropy data processing, Anal. Chem. 80 (2008) 6915-6927.

[59] T. Gruendling, D. Voll, M. Guilhaus, C. Barner-Kowollik, A perfect couple: PLP/SEC/ESI-MS for the accurate determination of propagation rate coefficients in free radical polymerization, Macromol. Chem. Phys. 211 (2010) 80-90.

[60] M. Dietrich, M. Glassner, T. Gruendling, C. Schmid, J. Falkenhagen, C. BarnerKowollik, Facile conversion of RAFT polymers into hydroxyl functional polymers:a detailed investigation of variable monomer and RAFT agent combinations, Polym. Chem. 1 (2010) 634-644.

[61] A. Feldermann, A.A. Toy, T.P. Davis, M.H. Stenzel, C. Barner-Kowollik, An in-depth analytical approach to the mechanism of the RAFT process in acrylate free radical polymerizations via coupled size exclusion chromatography-electrospray ionization mass spectrometry (SEC-ESI-MS), Polymer 46 (2005) 8448-8457.

[62] H. Barqawi, E. Ostas, B. Liu, J.-F. Carpentier, W.H. Binder, Multidimensional characterization of $\alpha, \omega$-telechelic poly( $\varepsilon$-caprolactone)s via online coupling of $2 \mathrm{D}$ chromatographic methods (LC/SEC) and ESI-TOF/MALDI-TOF-MS, Macromolecules 45 (2012) 9779-9790. 
[63] N. Pretorius, K. Rhode, J. Simpson, H. Pasch, Characterization of complex phthalic acid/propylene glycol based polyesters by the combination of $2 \mathrm{D}$ chromatography and MALDI-TOF mass spectrometry, Anal. Bioanal. Chem. 407 (2015) 217-230.

[64] H. Cölfen, M. Antonietti, New Developments in Polymer Analytics I (Advances in Polymer Science), Springer Berlin Heidelberg, 2000, p. 67-187.

[65] M. Hassellöv, G. Hulthe, B. Lyvén, G. Stenhagen, Electrospray mass spectrometry as online detector for low molecular weight polymer separations with flow field-flow fractionation, J. Liq. Chromatogr. Relat. Technol. 20 (1997) 2843-2856.

[66] G.R. Hilton, A.T. Jackson, K. Thalassinos, J.H. Scrivens, Structural analysis of synthetic polymer mixtures using ion mobility and tandem mass spectrometry, Anal. Chem. 80 (2008) 9720-9725.

[67] V. Scionti, B.C. Katzenmeyer, N. Solak, X. Li, C. Wesdemiotis, Interfacing multistage mass spectrometry with liquid chromatography or ion mobility separation for synthetic polymer analysis, Eur. J. Mass Spectrom. 18 (2012) 113-137.

[68]

http://www.waters.com/waters/library.htm?locale=en_US\&lid=134757057\&cid=5114 36 (last accessed 24.05.15).

[69] K. Kim, J. Lee, T. Chang, H. Kim, Characterization of polylactides with different stereoregularity using electrospray ionization ion mobility mass spectrometry, J. Am. Soc. Mass Spectrom. 25 (2014) 1771-1779.

[70] S. Trimpin, M. Plasencia, D. Isailovic, D.E. Clemmer, Resolving oligomers from fully grown polymers with IMS-MS, Anal. Chem. 79 (2007) 7965-7974.

[71] C. Barrere, F. Maire, C. Afonso, P. Giusti, Atmospheric solid analysis probe-ion mobility mass spectrometry of polypropylene, Anal. Chem. 84 (2012) 9349-9354. 
1492 Rapid analysis of polyester and polyethylene blends by ion mobility-mass spectrometry, 1493 Polym. Chem. 5 (2014) 3576-3582.

1494 [73] J. Song, C.H. Grün, R.M.A. Heeren, H.-G. Janssen, O.F. van den Brink, High1495 resolution ion mobility spectrometry-mass spectrometry on poly(methyl methacrylate), 1496 Angew. Chem. Int. Ed. 49 (2010) 10168-10171.

1497 [74] J.N. Hoskins, S. Trimpin, S.M. Grayson, Architectural differentiation of linear and 1498 cyclic polymeric isomers by ion mobility spectrometry-mass spectrometry, Macromolecules 44 (2011) 6915-6918.

[75] A.T. Jackson, K. Thalassinos, R.O. John, N. McGuire, D. Freeman, J.H. Scrivens, 1501 Characterisation of end groups in poly(2-hydroxyethyl methacrylate) by means of 1502 electrospray ionisation-mass spectrometry/mass spectrometry (ESI-MS/MS), Polymer 51 1503 (2010) 1418-1424.

1504

[76] K. Thalassinos, A.T. Jackson, J.P. Williams, G.R. Hilton, S.E. Slade, J.H. Scrivens, Novel software for the assignment of peaks from tandem mass spectrometry spectra of synthetic polymers, J. Am. Soc. Mass Spectrom. 18 (2007) 1324-1331.

[77] A. Jackson, S. Slade, K. Thalassinos, J. Scrivens, End-group characterisation of 1508 poly(propylene glycol)s by means of electrospray ionisation-tandem mass spectrometry (ESIMS/MS), Anal. Bioanal. Chem. 392 (2008) 643-650.

[78] J.P. Williams, G.R. Hilton, K. Thalassinos, A.T. Jackson, J.H. Scrivens, The rapid 1511 characterisation of poly(ethylene glycol) oligomers using desorption electrospray ionisation tandem mass spectrometry combined with novel product ion peak assignment software, Rapid Commun. Mass Spectrom. 21 (2007) 1693-1704. 
[79] A. Baumgaertel, K. Scheubert, B. Pietsch, K. Kempe, A.C. Crecelius, S. Böcker, U.S.

1515 Schubert, Analysis of different synthetic homopolymers by the use of a new calculation software for tandem mass spectra, Rapid Commun. Mass Spectrom. 25 (2011) 1765-1778. materials with emerging applications, Angew. Chem. Int. Ed. 51 (2012) 7898-7921.

[81] M.S. Montaudo, Mass spectra of copolymers, Mass Spectrom. Rev. 21 (2002) 108144.

[82] O.J. Houshia, C. Wilkins, Compositional analysis of the high molecular weight ethylene oxide propylene oxide copolymer by MALDI mass spectrometry, Int. J. Chem. 4 (2012) 14-23.

[83] G. Gallet, S. Carroccio, P. Rizzarelli, S. Karlsson, Thermal degradation of poly(ethylene oxide-propylene oxide-ethylene oxide) triblock copolymer: Comparative study by SEC/NMR, SEC/MALDI-TOF-MS and SPME/GC-MS, Polymer 43 (2002) 1081-1094. Polym. Degrad. Stab. 98 (2013) 356-360.

[85] R.P. Lattimer, M.J. Polce, C. Wesdemiotis, MALDI-MS analysis of pyrolysis products from a segmented polyurethane, J. Anal. Appl. Pyrolysis 48 (1998) 1-15.

[86] S.E. Whitson, C. Wesdemiotis, R.P. Lattimer, Characterization of polyurethane formulations by direct probe atmospheric pressure chemical ionization mass spectrometry, Rubber Chem. Technol. 83 (2010) 35-45.

1534 [87] S.E. Whitson, G. Erdodi, J.P. Kennedy, R.P. Lattimer, C. Wesdemiotis, Direct probe1535 atmospheric pressure chemical ionization mass spectrometry of cross-linked copolymers and 1536 copolymer blends, Anal. Chem. 80 (2008) 7778-7785. 
[88] A. Baumgaertel, E. Altuntaş, K. Kempe, A. Crecelius, U.S. Schubert, Characterization of different poly(2-oxazoline) block copolymers by MALDI-TOF MS/MS and ESI-Q-TOF MS/MS, J. Polym. Sci., Part A: Polym. Chem. 48 (2010) 5533-5540.

[89] A.C. Crecelius, C.R. Becer, K. Knop, U.S. Schubert, Block length determination of the block copolymer mPEG-b-PS using MALDI-TOF MS/MS, J. Polym. Sci., Part A: Polym. Chem. 48 (2010) 4375-4384.

[90] B.A. Cerda, D.M. Horn, K. Breuker, F.W. McLafferty, Sequencing of specific copolymer oligomers by electron-capture-dissociation mass spectrometry, J. Am. Chem. Soc. 124 (2002) 9287-9291.

[91] S.M. Miladinović, C.J. Kaeser, M.M. Knust, C.L. Wilkins, Tandem Fourier transform mass spectrometry of block and random copolymers, Int. J. Mass Spectrom. 301 (2011) 184194.

[92] E.R. Kaal, M. Kurano, M. Geißler, H.-G. Janssen, Hyphenation of aqueous liquid chromatography to pyrolysis-gas chromatography and mass spectrometry for the comprehensive characterization of water-soluble polymers, J. Chromatogr. A 1186 (2008) 222-227.

[93] S.M. van Leeuwen, B. Tan, D.W. Grijpma, J. Feijen, U. Karst, Characterization of the chemical composition of a block copolymer by liquid chromatography/mass spectrometry using atmospheric pressure chemical ionization and electrospray ionization, Rapid Commun. Mass Spectrom. 21 (2007) 2629-2637.

[94] H. Hayen, A.A. Deschamps, D.W. Grijpma, J. Feijen, U. Karst, Liquid chromatographic-mass spectrometric studies on the in vitro degradation of a poly(ether ester) block copolymer, J. Chromatogr. A 1029 (2004) 29-36.

[95] M. Girod, T.N.T. Phan, L. Charles, Tuning block copolymer structural information by adjusting salt concentration in liquid chromatography at critical conditions coupled with 
electrospray tandem mass spectrometry, Rapid Commun. Mass Spectrom. 23 (2009) 14761482.

[96] N. Fandrich, J. Falkenhagen, S.M. Weidner, B. Staal, A.F. Thünemann, A. Laschewsky, Characterization of new amphiphilic block copolymers of N-vinylpyrrolidone and vinyl acetate, 2 - chromatographic separation and analysis by MALDI-TOF and FT-IR coupling, Macromol. Chem. Phys. 211 (2010) 1678-1688.

[97] A. Baumgaertel, C. Weber, N. Fritz, G. Festag, E. Altuntaş, K. Kempe, R. Hoogenboom, U.S. Schubert, Characterization of poly(2-oxazoline) homo- and copolymers by liquid chromatography at critical conditions, J. Chromatogr. A 1218 (2011) 8370-8378.

[98] S.M. Weidner, J. Falkenhagen, LC-MALDI-TOF imaging MS: A new approach in combining chromatography and mass spectrometry of copolymers, Anal. Chem. 83 (2011) 9153-9158.

[99] H. Barqawi, M. Schulz, A. Olubummo, V. Saurland, W.H. Binder, 2D-LC/SEC(MALDI-TOF)-MS characterization of symmetric and nonsymmetric biocompatible PEOmPIB-PEOn block copolymers, Macromolecules 46 (2013) 7638-7649.

[100] M.I. Malik, B. Trathnigg, R. Saf, Characterization of ethylene oxide-propylene oxide block copolymers by combination of different chromatographic techniques and matrixassisted laser desorption ionization time-of-flight mass spectroscopy, J. Chromatogr. A 1216 (2009) 6627-6635.

[101] S.M. Weidner, J. Falkenhagen, I. Bressler, Copolymer composition determined by LCMALDI-TOF MS coupling and "MassChrom2D" data analysis, Macromol. Chem. Phys. 213 (2012) 2404-2411.

[102] S. Huijser, B.B.P. Staal, J. Huang, R. Duchateau, C.E. Koning, Chemical composition and topology of poly(lactide-co-glycolide) revealed by pushing MALDI-TOF MS to its limit, Angew. Chem. Int. Ed. 118 (2006) 4210-4214. 
[103] G. Vivó-Truyols, B. Staal, P.J. Schoenmakers, Strip-based regression: A method to 1588 obtain comprehensive co-polymer architectures from matrix-assisted laser desorption ionisation-mass spectrometry data, J. Chromatogr. A 1217 (2010) 4150-4159.

[104] R.X.E. Willemse, B.B.P. Staal, E.H.D. Donkers, A.M. van Herk, Copolymer (2004) 5717-5723.

1593 [105] G. Wilczek-Vera, P.O. Danis, A. Eisenberg, Individual block length distributions of block copolymers of polystyrene-block-poly( $\alpha$-methylstyrene) by MALDI/TOF mass spectrometry, Macromolecules 29 (1996) 4036-4044.

[106] A. Chojnacka, K. Kempe, H.C. van de Ven, C. Englert, R. Hoogenboom, U.S.

Schubert, H.-G. Janssen, P. Schoenmakers, Molar mass, chemical-composition, and 1598 functionality-type distributions of poly(2-oxazoline)s revealed by a variety of separation 1599 techniques, J. Chromatogr. A 1265 (2012) 123-132.

[107] A.M. Yol, J. Janoski, R.P. Quirk, C. Wesdemiotis, Sequence analysis of styrenic copolymers by tandem mass spectrometry, Anal. Chem. 86 (2014) 9576-9582.

1602 [108] S. Huijser, G.D. Mooiweer, R. van der Hofstad, B.B.P. Staal, J. Feenstra, A.M. van Herk, C.E. Koning, R. Duchateau, Reactivity ratios of comonomers from a single MALDIToF-MS measurement at one feed composition, Macromolecules 45 (2012) 4500-4510.

[109] S. Trimpin, D.E. Clemmer, Ion mobility spectrometry/mass spectrometry snapshots for assessing the molecular compositions of complex polymeric systems, Anal. Chem. 80 (2008) $16079073-9083$.

1608 [110] M.S. Engler, S. Crotty, M.J. Barthel, C. Pietsch, K. Knop, U.S. Schubert, S. Böcker, 1609 COCONUT - an efficient tool for estimating copolymer compositions from mass spectra, Anal. Chem. 87 (2015) 5223-5231. 
[111] R. Gutzler, M. Smulders, R.F.M. Lange, The role of synthetic pharmaceutical polymer excipients in oral dosage forms - poly(ethylene oxide)-graft-poly(vinyl alcohol) copolymers in tablet coatings, Macromol. Symp. 225 (2005) 81-94.

[112] M. Adler, F. Rittig, S. Becker, H. Pasch, Multidimensional chromatographic and hyphenated techniques for hydrophilic copolymers, 1, Macromol. Chem. Phys. 206 (2005) 2269-2277.

[113] A.M. Yol, D.E. Dabney, S.-F. Wang, B.A. Laurent, M.D. Foster, R.P. Quirk, S.M. Grayson, C. Wesdemiotis, Differentiation of linear and cyclic polymer architectures by MALDI tandem mass spectrometry (MALDI-MS2), J. Am. Soc. Mass Spectrom. 24 (2013) $74-82$.

[114] J. Maslinska-Solich, E. Kudrej-Gibas, Electrospray mass spectrometry determination of poly(oxazolidine acetal), Rapid Commun. Mass Spectrom. 17 (2003) 1769-1774.

[115] J. Maslinska-Solich, E. Gibas, Electrospray mass spectrometry studies of macromolecules containing 1,3-oxazolidine moieties, Rapid Commun. Mass Spectrom. 18 (2004) 2619-2628.

[116] O. Wachsen, K.H. Reichert, R.P. Krueger, H. Much, G. Schulz, Thermal decomposition of biodegradable polyesters. III. Studies on the mechanisms of thermal degradation of oligo-L-lactide using SEC, LACCC and MALDI-TOF-MS, Polym. Degrad. Stab. 55 (1997) 225-231.

[117] L.A. McDonnell, P.J. Derrick, B.B. Powell, P. Double, Sustained off-resonance irradiation collision-induced dissociation of linear, substituted and cyclic polyesters using a 9.4 T Fourier transform ion cyclotron resonance mass spectrometer, Eur. J. Mass Spectrom. 9 (2003) 117-128. 
[118] S.-F. Wang, X. Li, R.L. Agapov, C. Wesdemiotis, M.D. Foster, Probing surface concentration of cyclic/linear blend films using surface layer MALDI-TOF mass spectrometry, ACS Macro Lett. 1 (2012) 1024-1027.

[119] X. Li, L. Guo, M. Casiano-Maldonado, D. Zhang, C. Wesdemiotis, Top-down multidimensional mass spectrometry methods for synthetic polymer analysis, Macromolecules 44 (2011) 4555-4564.

[120] H. Chaffey-Millar, G. Hart-Smith, C. Barner-Kowollik, Living star polymer formation (RAFT) studied via electrospray ionization mass spectrometry, J. Polym. Sci., Part A: Polym. Chem. 46 (2008) 1873-1892.

[121] A.A. Toy, P. Vana, T.P. Davis, C. Barner-Kowollik, Reversible addition fragmentation chain transfer (RAFT) polymerization of methyl acrylate: Detailed structural investigation via coupled size exclusion chromatography-electrospray ionization mass spectrometry (SEC-ESIMS), Macromolecules 37 (2004) 744-751.

[122] J. Allgaier, K. Martin, H.J. Raeder, K. Muellen, Many-arm star polymers synthesized using chlorosilane linking agents: Their structural quality concerning arm number and polydispersity, Macromolecules 32 (1999) 3190-3194.

[123] G. Wang, X. Fan, B. Hu, Y. Zhang, J. Huang, Synthesis of eight-shaped poly(ethylene oxide) by the combination of glaser coupling with ring-opening polymerization, Macromol. Rapid Commun. 32 (2011) 1658-1663.

[124] D. Morsa, T. Defize, D. Dehareng, C. Jérôme, E. De Pauw, Polymer topology revealed by ion mobility coupled with mass spectrometry, Anal. Chem. 86 (2014) 9693-9700.

[125] Y. Li, B. Zhang, J.N. Hoskins, S.M. Grayson, Synthesis, purification, and characterization of "perfect" star polymers via "Click" coupling, J. Polym. Sci., Part A: Polym. Chem. 50 (2012) 1086-1101. 
[126] T. Rudolph, S. Crotty, M. von der Lühe, D. Pretzel, U.S. Schubert, F.H. Schacher, Synthesis and solution properties of double hydrophilic poly(ethylene oxide)-block-poly(2ethyl-2-oxazoline) (PEO-b-PEtOx) star block copolymers, Polymers 5 (2013) 1081-1101.

[127] D. Yu, N. Vladimirov, J.M.J. Frechet, MALDI-TOF in the characterizations of dendritic-linear block copolymers and stars, Macromolecules 32 (1999) 5186-5192.

[128] L.P. Tolic, G.A. Anderson, R.D. Smith, H.M. Brothers, II, R. Spindler, D.A. Tomalia, Electrospray ionization Fourier transform ion cyclotron resonance mass spectrometric characterization of high molecular mass Starburst dendrimers, Int. J. Mass Spectrom. Ion Processes 165/166 (1997) 405-418.

[129] M. He, S.A. McLuckey, Tandem mass spectrometry of half-generation PAMAM dendrimer anions, Rapid Commun. Mass Spectrom. 18 (2004) 960-972.

[130] S. Lee, S.Y. Han, T.G. Lee, G. Chung, D. Lee, H.B. Oh, Observation of pronounced $\mathrm{b}, \mathrm{y}$ cleavages in the electron capture dissociation mass spectrometry of polyamidoamine (PAMAM) dendrimer ions with amide functionalities, J. Am. Soc. Mass Spectrom. 17 (2006) 536-543.

[131] F. Maire, G. Coadou, L. Cravello, C.M. Lange, Traveling wave ion mobility mass spectrometry study of low generation polyamidoamine dendrimers, J. Am. Soc. Mass Spectrom. 24 (2013) 238-248.

[132] A. Tintaru, S. Pricl, L. Denbigh, X. Liu, L. Peng, L. Charles, Conformational changes of small PAMAM dendrimers as a function of their charge state: A combined electrospray mass spectrometry, traveling-wave ion mobility and molecular modeling study, Int. J. Mass Spectrom. 354-355 (2013) 235-241.

[133] H. So, J. Lee, S.Y. Han, H.B. Oh, MALDI in-source decay mass spectrometry of polyamidoamine dendrimers, J. Am. Soc. Mass Spectrom. 23 (2012) 1821-1825. 
[134] A. Adhiya, C. Wesdemiotis, Poly(propylene imine) dendrimer conformations in the gas phase: A tandem mass spectrometry study, Int. J. Mass Spectrom. 214 (2002) 75-88.

[135] J.-W. Weener, J.L.J. van Dongen, J.C. Hummelen, E.W. Meijer, Fragmentation studies of poly(propylene imine) dendrimers in the gas-phase by using electrospray ionization mass spectrometry (ESI-MS), Polym. Mater. Sci. Eng. 77 (1997) 147-148.

[136] J.-W. Weener, J.L.J. van Dongen, E.W. Meijer, Electrospray mass spectrometry studies of poly(propylene imine) dendrimers: Probing reactivity in the gas phase, J. Am. Chem. Soc. 121 (1999) 10346-10355.

[137] M.A.C. Broeren, J.L.J. van Dongen, M. Pittelkow, J.B. Christensen, M.H.P. van Genderen, E.W. Meijer, Multivalency in the gas phase: The study of dendritic aggregates by mass spectrometry, Angew. Chem., Int. Ed. 43 (2004) 3557-3562.

[138] S. van der Wal, Y. Mengerink, J.C. Brackman, E.M.M. de Brabander, C.M. Jeronimus-Stratingh, A.P. Bruins, Compositional analysis of nitrile terminated poly(propylene imine) dendrimers by high-performance liquid chromatography combined with electrospray mass spectrometry, J. Chromatogr. A 825 (1998) 135-147.

[139] L. Ye, Z.-G. Feng, Y.-M. Zhao, F. Wu, S. Chen, G.-Q. Wang, Synthesis and application as polymer electrolyte of hyperbranched polyether made by cationic ring-opening polymerization of 3-\{2-[2-(2-hydroxyethoxy)ethoxy]ethoxymethyl\}-3'-methyl-oxetane, J. Polym. Sci., Part A: Polym. Chem. 44 (2006) 3650-3665.

[140] R.L.C. Lau, T.W.D. Chan, I.Y.K. Chan, H.-F. Chow, Fourier transform ion cyclotron resonance studies of terpyridine-based polyether dendrimers and their iron(II) metallocomplexes by liquid secondary ion mass spectrometry, Eur. Mass Spectrom. 1 (1995) 371-380. 
[141] G. Coullerez, H.J. Mathieu, S. Lundmark, M. Malkoch, H. Magnusson, A. Hult,

Cationization of dendritic macromolecule adsorbates on metals studied by time-of-flight secondary ion mass spectrometry, Surf. Interface Anal. 35 (2003) 682-692.

1708

[142] A.J. Clark, A.A. Coleshill, D.M. Haddleton, S.J. Isles, H.S. Sahota, P.C. Taylor,

Changes in the homogeneity of poly(arylester) dendrimers during the matrix-assisted laser desorption/ionization mass spectrometry experiment, Eur. Mass Spectrom. 5 (1999) 273-277.

[143] H. Sahota, P.M. Lloyd, S.G. Yeates, P.J. Derrick, P.C. Taylor, D.M. Haddleton, Characterization of aromatic polyester dendrimers by matrix-assisted laser desorption ionization mass spectrometry, J. Chem. Soc., Chem. Commun. (1994) 2445-2446.

Fokkens, N.M.M. Nibbering, In-source decay of hyperbranched polyesteramides in matrixassisted laser desorption/ionization time-of-flight mass spectrometry, J. Am. Soc. Mass Spectrom. 11 (2000) 218-227.

[145] S. Koster, M.C. Duursma, J.J. Boon, R.M.A. Heeren, S. Ingemann, R.A.T.M. van Benthem, C.G. de Koster, Electron capture and collisionally activated dissociation mass spectrometry of doubly charged hyperbranched poly(ester-amide)s, J. Am. Soc. Mass Spectrom. 14 (2003) 332-341.

[146] W. Hayes, A.W. Freeman, J.M.J. Frechet, Matrix-assisted laser desorption ionization mass spectrometric analysis of aromatic polyether dendritic systems, Polym. Mater. Sci. Eng. 77 (1997) 136-137.

1725 [147] N. Yamazaki, I. Washio, Y. Shibasaki, M. Ueda, Facile synthesis of poly(phenylene1726 ether) dendrimers from unprotected AB2-building block using thionyl chloride as a condensing agent, Polym. Prepr. 46 (2005) 645-646. 
1728 [148] J.W. Leon, J.M.J. Frechet, Analysis of aromatic polyether dendrimers and dendrimer-

1729 linear block copolymers by matrix-assisted laser desorption ionization mass spectrometry, 1730 Polym. Bull. 35 (1995) 449-455.

1731 [149] I.A. Mowat, R.J. Donovan, M. Bruce, W.J. Feast, N.M. Stainton, Matrix assisted laser 1732 desorption/ionization mass spectrometry of aryl ester dendrimers, Eur. Mass Spectrom. 4 $1733 \quad$ (1998) 451-458.

1734 [150] A.P. Gies, M. Kliman, J.A. McLean, D.M. Hercules, Characterization of branching in 1735 aramid polymers studied by MALDI-ion mobility/mass spectrometry, Macromolecules 41 1736 (2008) 8299-8301.

1737 [151] K. Im, S. Park, D. Cho, T. Chang, K. Lee, N. Choi, HPLC and MALDI-TOF MS 1738 analysis of highly branched polystyrene: resolution enhancement by branching, Anal. Chem. $173976(2004) 2638-2642$.

1740 [152] X. Liu, L.R. Cool, K. Lin, A.M. Kasko, C. Wesdemiotis, Tandem mass spectrometry 1741 and ion mobility mass spectrometry for the analysis of molecular sequence and architecture of 1742 hyperbranched glycopolymers, Analyst 140 (2015) 1182-1191. 
1752 Graphical Abstract

1753

1754

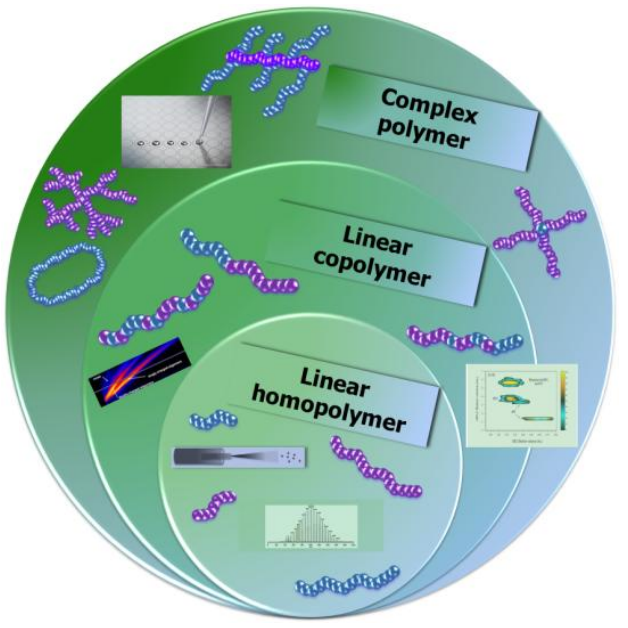

1755

1756

1757

1758

1759

1760

1761

1762 
1763 Sarah Crotty was born in Ambilly (France) in 1987. She studied chemistry and analytical 1764 chemistry in Loughborough (B.Sc. in 2010) and Warwick (M.Sc. in 2011) (both UK). She 1765 worked in India for one year in $R \& D$ investigating the synthesis and characterization of 1766 potential drugs for tropical diseases. She joined in 2012 the research group of Prof. Ulrich S. 1767 Schubert as a Ph.D. student at the Friedrich Schiller University Jena. Her research focuses on 1768 the characterization of synthetic polymers by the use of diverse techniques 2D-LC, liquid 1769 adsorption chromatography at critical conditions (LACCC), spotting, mass spectrometry (MALDI-, ESI- \& MS/MS) and software algorithm.

Selim Gerişlioğlu was born in Izmir (Turkey) in 1989. He studied chemistry at Hacettepe University (B.Sc. in 2010) (Ankara, Turkey). He completed his M.Sc. degree on physical chemistry in 2012 at Hacettepe University where he investigated ionization mechanisms in MALDI-MS. He moved to Akron, USA and joined the research group of Prof. Chrys Wesdemiotis as a Ph.D. student at The University of Akron in 2013. His current research focuses on investigation of fragmentation mechanisms and structural characterization of copolymers by utilizing different tandem mass spectrometric techniques such as electron transfer dissociation and collisionally activated dissociation.

Kevin Endres was born in Addison, IL (U.S.) in 1992. He studied Specialized Chemistry at the University of Illinois at Urbana-Champaign (B.Sc. in 2014), and conducted synthetic research on self-assembling nanoparticles for potential self-healing applications. He joined the research group of Prof. Chrys Wesdemiotis as a Ph.D. student at the University of Akron. His research focuses on the characterization of synthetic polymers with mass spectrometry (ESI and MALDI), polymer and supramolecular structures with IM-ESI-MS, and polymer blended 
Chrys Wesdemiotis was born in Thessaloniki (Greece) in 1952. He completed his B.Sc.,

1789

1790

1791

1792

1793

1794

1795

1796

1797

1798

1799

1800

1801

1802

1803

1804

1805

1806

1807

1808

1809

1810

1811

1812

M.Sc. and Ph.D. at Technische Universität Berlin (1979). After a postdoctoral fellowship with Fred W. McLafferty at Cornell University (1980) and military service in Greece (1981-1983), he returned to Cornell as senior research associate (1983-1989). In 1989, he joined the University of Akron, where he currently is Distinguished Professor of Chemistry, Polymer Science, and Integrated Bioscience. Wesdemiotis is AAAS fellow, Editor of the European Journal of Mass Spectrometry, and member of the Editorial Boards of Mass Spectrometry Reviews, International Journal of Mass Spectrometry, and the Journal of the American Society for Mass Spectrometry. Research in the Wesdemiotis group focuses on the development and applications of multidimensional mass spectrometry methodologies for the characterization of new synthetic polymers and polymer-biomolecule interfaces.

Ulrich S. Schubert was born in Tubingen (Germany) in 1969. He studied chemistry in Frankfurt and Bayreuth (both Germany) as well as in Richmond (USA). His Ph.D. studies were performed at the Universities of Bayreuth and South Florida/Tampa (USA). After a postdoctoral training with Jean-Marie Lehn at the University of Strasbourg (France), he moved to the TU Munich (Germany) and obtained his Habilitation in 1999. From 1999 to 2000 he was Professor at the Center for NanoScience, University of Munich (Germany), and from 2000 to 2007 Full-Professor at Eindhoven University of Technology (The Netherlands). Currently, he holds a chair at the Friedrich Schiller University Jena with research interest in nanoparticle systems as sensor and drug delivery devices, supramolecular chemistry, inkjet printing of polymers, polymers for energy storage, and self-healing materials. 
1813



1814

1815

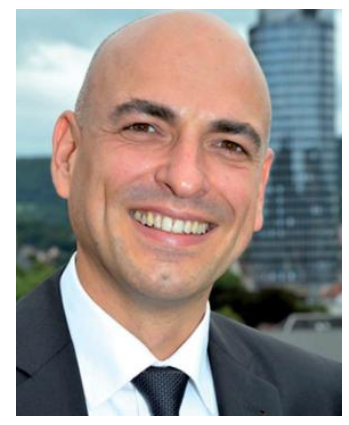

1816

1817

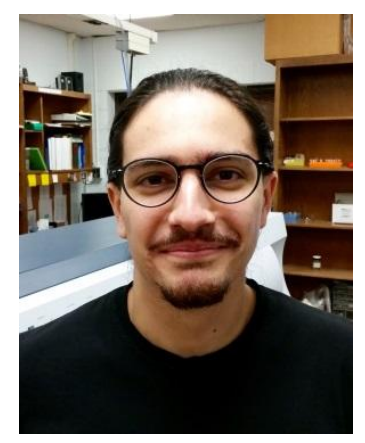

1818

1819

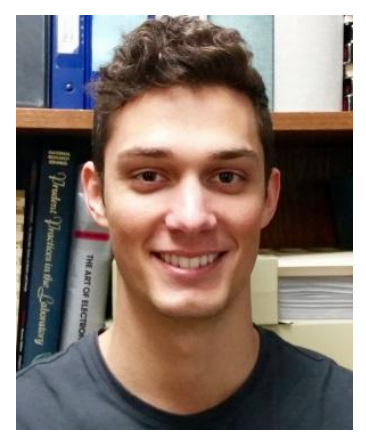

1820 
1821

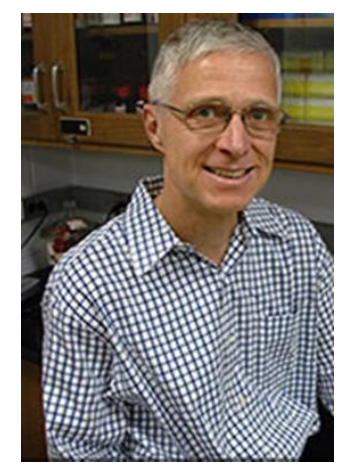

